\title{
Bedrohungsempfinden als Quelle gruppenbezogener Vorurteile durch Religionen in einer heterogenen Stadtgesellschaft. Analysen des Berlinmonitors
}

\author{
Gert Pickel (D) Kazim Celik · Julia Schuler • Oliver Decker
}

Online publiziert: 7. Mai 2020

(C) Der/die Autor(en) 2020

Zusammenfassung Gruppenbezogene Vorurteilen und Ressentiments sind eine Belastung für die liberale die Demokratie. Sie reflektieren häufig eine Ablehnung und eine Angst vor gesellschaftlicher Veränderung. Dabei scheinen religiöse Gruppen besonders stark betroffen, nimmt man die vielfältigen öffentlichen Diskussionen zu antimuslimischen Ressentiments und Antisemitismus zur Kenntnis. Gleichzeitig kann Religiosität auch Vorurteile hemmen, bestärken - oder gar hervorbringen. Im vorliegenden Beitrag werden der Daten des 2019 erhobenen Berlin-Monitors mit dem Ziel mögliche Zusammenhänge zwischen einer differenzierter erfassten Religiosität und Vorurteilen bzw. Ressentiments zu untersuchen. Die Wirkung von Religiosität ist zeigeteilt: Religiös dogmatische bis fundamentalistische Menschen neigen eher zu Vorurteilen als andere Bevölkerungsgruppen. Liberale, oft sozial engagierte Christ*innen oder auch Muslim*innen weisen dagegen weniger Vorurteile auf. Dabei finden sich speziell für den Antisemitismus zwei besonders anfällige Gruppen: Rechtorientierte Menschen und dogmatische Muslim*innen. Dies kann auch in Berlin, wo die Bürger*innen im Vergleich zum Bundesgebiet toleranter sind, nachgewiesen werden.

Schlüsselwörter Religion · Bedrohung · Politische Kultur · Vorurteile · Berlin

\footnotetext{
Der vorliegende Artikel beruht in Teilen auf Forschungstätigkeit, die in der Kollegforschergruppe „Multiple Secularities - Beyond the West, Beyond Modernities“ an der Universität Leipzig durchgeführt wurde. Für die dort gewährte Hilfe und Möglichkeiten danke ich an dieser Stelle. Die Kollegforschergruppe wird durch die Deutsche Forschungsgesellschaft (DFG) gefördert. Für weitere Informationen: www.multiple.secularities.de.
} 


\title{
Perception of threat as a source of group-related prejudices by religions in a heterogeneous urban society. Analyses of the Berlin Monitor
}

\begin{abstract}
Group-related prejudices and resentments are a burden on liberal democracy. They often reflect a rejection and a fear of social change. Religious groups seem to be particularly affected, if one takes note of the manifold public discussions on anti-Muslim resentment and anti-Semitism. At the same time, religiosity can also inhibit, reinforce-or even produce-prejudices. This article uses data from the 2019 Berlin-Monitor to investigate possible connections between a more differentiated assessment of religiosity and prejudices or resentments. The effect of religiosity is divided into two parts: Religiously dogmatic to fundamentalist people are more prone to prejudice than other population groups. Liberal, often socially committed Christians or also Muslims, on the other hand, have fewer prejudices. Two groups are particularly vulnerable to anti-Semitism: Rights-oriented people and dogmatic Muslims. This can also be demonstrated in Berlin, where the citizens are more tolerant compared to the rest of Germany.
\end{abstract}

Keywords Religion · Threat · Political culture $\cdot$ Prejudice $\cdot$ Berlin

\section{Einleitung - Angst als Quelle für Vorurteile?}

Man benötigt eigentlich gar nicht die jüngsten antisemitischen Anschläge in Halle, um die Relevanz und Gefährlichkeit von gruppenbezogenen Vorurteilen und Ressentiments für die Demokratie zu verstehen. ${ }^{1}$ So haben sich diese in der deutschen Geschichte zeitlich versetzt immer wieder in Gewaltakten niedergeschlagen, ganz abgesehen von täglichem Alltagsrassismus, Diskriminierungen und Gesprächen, privaten und öffentlichen Äußerungen, in denen ihre tiefe Verwurzelung in der Gesellschaft zu Tage tritt. ${ }^{2}$ Auf Gruppen bezogene, pauschalisierende, Vorurteile reflektieren häufig eine Ablehnung von gesellschaftlicher Pluralität, die emotional, meist aus Angst begründet ist (Adorno 1973, S. 287-297; Tajfel 1982). Da die heutigen europäischen Gesellschaften durch weitreichende Prozesse der Pluralisierung und gesellschaftlichen Differenzierung geprägt sind, weitet sich die Zahl sozialer Gruppen aus, die Ziel sozialer Abwertung und von Vorurteilen werden können. ${ }^{3}$ Innerhalb

\footnotetext{
1 Wir danken den beiden Gutachter*innen für ihre zielführenden und ausführlichen Hinweise, die noch einmal in erheblichem Maß zur Verbesserung des Textes beigetragen haben.

2 In der vorliegenden Einleitung werden die Begriffe gruppenbezogene Vorurteile, wie Ressentiments nebeneinander verwendet, obwohl sie Unterschiedliches beschreiben. Ihr Definition und Differenzierung erfolgt in Abschn. 2 des Beitrags. In der Regel werden wir allerdings in diesem Aufsatz die Bezeichnung Vorurteile im Sinne gruppenbezogener Vorurteile verwenden, um Irritationen zu vermeiden. Dem ungeachtet muss von Fall zu Fall unterschieden werden, ob man die gemessenen Haltungen als Ressentiments oder als Vorurteile einstuft.

3 Dies unterscheidet moderne Gesellschaften in ihrer Heterogenität traditionell von homogen gedachten Gemeinschaften (Tönnies 2010). Gleichzeitig wohnen auch Gesellschaften immer ihre Mitglieder verbindende Elemente inne. Der politischen Kulturforschung nach, ist die gemeinsame Anerkennung der politischen Gemeinschaft seitens aller ihrer Mitglieder von essenzieller Bedeutung für die Stabilität des politischen Systems und den gesellschaftlichen Frieden (Almond und Verba 1963; Easton 1975; Pickel
} 
dieser Pluralisierung, die eine Pluralisierung der Vorurteile beinhaltet, sind in den letzten Jahren soziale Gruppen, die über ihre Religionszugehörigkeit definiert werden, stärker ins Zentrum der Vorurteilsforschung gerückt. Wohlmöglich bietet sich gerade heute, in zunehmend säkularer werdenden Gesellschaften, die Zugehörigkeit zu bestimmten religiösen Gemeinschaften als guter Referenzpunkt für gruppenbezogene Vorurteile an. Neben dem Eindruck, dass allein die Unterschiedlichkeit zu dem, was viele Bürger*innen als ,normale Bürger*innen“ ansehen, ausreicht, um massive Abgrenzungen und Abwertungsprozesse einzuleiten oder aufrecht zu erhalten, strahlen gerade religiöse Gruppen eine Aura von (nicht mehr bekannter) kultureller Bedrohung aus, die anderen sozialen Gruppen nicht in gleichem Maße innewohnt. Als Beispiele zu nennen sei nur die hartnäckige Existenz von Antisemitismus in vielen europäischen Ländern und die jüngste Verbreitung antimuslimischer Vorurteile (Beller 2015; Blume 2019; Decker et al. 2016, S. 95-136; Decker und Brähler 2018; Helbling 2012; Pickel 2018; Salzborn 2014; Strabac und Listhaug 2007; Yendell 2013; Zick et al. 2016, 2019).

Nun stehen Vorurteile gegenüber Religionsgemeinschaften nicht alleine oder singular. Es gibt mannigfaltige Hinweise auf eine enge Verwobenheit von gruppenbezogenen Vorurteilen, wie sie z.B. das Konzept der Gruppenbezogenen Menschenfeindlichkeit herausstellt (Heitmeyer 2002, 2010; Zick et al. 2019, S. 55-64; Zick und Küpper 2009). Zudem existieren Hinweise auf eine Verzahnung von Vorurteilen in Bezug auf unter Vorurteilen leidenden sozialen Gruppen, bei Personen, welche sich intersektionaler Abwertung ausgesetzt sehen. Diese Überlegungen führen uns in diesem Aufsatz zu vier Fragen: ${ }^{4}$

1. Wie verteilen sich gruppenbezogene Vorurteile in heterogenen Gesellschaften?

2. Sind religiöse Gruppen wirklich stärker davon betroffen als andere soziale Gruppen?

3. Welche Rolle spielt ein Gefühl der Bedrohung durch eine sozialen Gruppe für gruppenbezogene Vorurteile ihr gegenüber?

4. Welche Folgen besitzen diese Bedrohungsgefühle und Vorurteile für die politische Kultur - oder: Sind es gerade Vorurteile gegenüber einer als gefährlich eingestuften religiösen Sozialgruppe, welche derzeit Rechtspopulisten zu ihren Erfolgen verhelfen?

Unsere Thesen sind: (1) Soziale Gruppen die als bedrohlich und gefährlich wahrgenommen werden, sind stärker von Vorurteilen und Ressentiments betroffen als andere soziale Gruppen. (2) Religiöse Sozialgruppen werden als besonders gefährlich eingeschätzt, da sie den Eindruck hoher kultureller Fremdheit vermitteln und entsprechende (kollektive) Ängste hervorrufen. (3) Die Markierung einer gefährlichen Fremdgruppe - über ihre Zugehörigkeit zu einer Religionsgemeinschaft - ist

und Pickel 2006, 2020a, b). Im strukturfunktionalistischen Kontext ist die soziale Integration der Gesellschaftsmitglieder unerlässlich für ein funktionierendes politisches System, während Konflikte unter den verschiedenen Teilen der Gesellschaft dysfunktional sind (Parsons 1951; Lipset 1981).

4 Um diesen Aufsatz stringent zu halten, haben wir weitgehend auf die Untersuchung der Wirkungen von Religiosität auf Vorurteile verzichtet. Die Untersuchung dieser Effekte ist ein Aufsatz für sich. Im vorliegenden Aufsatz liegt der Fokus auf der differenzierten Betrachtung der Haltungen gegenüber religiösen Bezugsgruppen. 
eine für Rechtspopulisten erfolgsträchtige Strategie (gerade auch über den Einsatz von Verschwörungstheorien). Unsere vorausgehende Annahme ist, dass Bedrohungsgefühle kausal Vorurteilen vorgeschaltet sind. Wir gehen davon aus, dass Ängste Vorurteile hervorbringen oder bestärken, ohne dabei allerdings in Abrede zu stellen, dass reziproke Prozesse Folge sein können oder gar vorangehen.

Als Referenzbeispiel wählen wir die Berliner Stadtbevölkerung. Sie weist eine hohe ethnische, religiöse und soziale Heterogenität auf und ist starken Dynamiken der Zuwanderung und Mobilität ausgesetzt. In gewisser Hinsicht kann sie als Paradefall bzw. Representative oder gar Crucial Case (Muno 2009, S. 117) für eine moderne Stadtgesellschaft angesehen werden. Wir gehen davon aus, dass die Bedingungen für positive wie negative Wirkungen von Heterogenität in Berlin stark ausgeprägt sind. So müssten angesichts der dynamischen Pluralisierung in Berlin Bedrohungswahrnehmungen mit Fokus auf Zuwanderung stärker ausfallen, wie ihnen die dadurch erhöhten Kontaktmöglichkeiten wiederum entgegenwirken. Entsprechend erscheint uns das im bundesdeutschen Vergleich als besonders multikulturell und offen eingestufte Berlin als guter Testfall für die aufgestellten Thesen.

Entsprechend werden aufbauend auf Überlegungen der Vorurteilsforschung, sozialpsychologischen Bedrohungstheorien (Integrated Threat Theory) (Stephan und Renfro 2002), der Autoritarismusforschung (Adorno 1973) und der Kontakthypothese (Tajfel 1982) im folgenden Beitrag aktuelle Daten zur Berliner Stadtgesellschaft quantitativ-empirisch untersucht. Als Quelle für die vorgestellten Überlegungen verwenden wir die Anfang 2019 erhobenen Daten des Berlin-Monitors 2019 (Pickel et al. 2019). Abgesehen von ihrer Aktualität, enthalten die Daten verschiedene gruppenbezogenen Vorurteile, die miteinander kontrastiert werden können. Zudem wurden unter den Berliner*innen Bedrohungsgefühle hinsichtlich von Mitgliedern von Religionsgemeinschaften, wie verschiedene potentielle Erklärungsfaktoren für gruppenbezogene Vorurteile erfasst.

\section{Konzeptionelle Überlegungen}

\subsection{Gruppenbezogene Vorurteile, Menschenfeindlichkeit und Ressentiments - Begriffe}

Die Unterscheidung zwischen gruppenbezogenen Vorurteilen und Ressentiments ist nicht ganz einfach. Gruppenbezogene Vorurteile, ein Begriff aus der sozialpsychologischen Vorurteilsforschung, bildet Abgrenzungen und Kategorisierungen sozialer Gruppen aufgrund wahrgenommener und zugeschriebener Gruppenmerkmale ab. Er schließt damit an die zentralen Überlegungen der Vorurteilsforschung an (Allport 1979; Pelinka et al. 2009). Dabei wird ein Vorurteil als ,eine Antipathie, die sich auf eine fehlerhafte und starre Verallgemeinerung gründet" (Klein 2014, S. 29) und in gruppenbezogener Erweiterung als ,Einstellung oder Orientierung gegenüber einer Gruppe (bzw. ihren Mitgliedern), die sie direkt oder indirekt abwertet, verstanden. Grund ist oft Eigeninteresse oder der Nutzen der eigenen Gruppe“ (Jonas et al. 2014, S. 509; auch Allport 1979, S. 6). Vorurteile können grundsätzlich positiv wie negativ sein. Z. B. Chinesen sind immer sehr disziplinierte und hart arbeitende Men- 
schen, Sinti und Roma neigen per se zu Kriminalität. Diese Vorurteile beruhen nicht zwingend auf Erfahrungen, sondern können sekundär (z. B. über Hörensagen, Medienberichte) konstruiert sein. Gelegentlich fehlen Erfahrungen gänzlich. Vorurteile können sich auf einzelne Personen richten, aber auch auf soziale Gruppen, denen man Eigenschaften zuweist - und sie deswegen ablehnt. Neben die Fremdkategorisierung tritt somit eine affektive Ablehnung, der oft auch in Abwertung Ausdruck verliehen wird. Ressentiments greifen in der Regel tiefer als Vorurteile und gründen in einer, fast immer auf Unterlegenheitsgefühlen beruhenden, unbewussten Abneigung, bzw. Projektionen des eigenen Scheiterns auf den Anderen (Decker et al. 2018, S. 183). ${ }^{5}$ Aspekte des Gefühls eines persönlichen Zurückgesetztseins spielen genauso eine Rolle für Distanz und Abwertung, wie unterdrückte Schuldgefühle, die man ablegen möchte und deren Existenz man der abgelehnten Gruppe zuschreibt. Anders als Vorurteile sind Ressentiments nie positiv und drücken sich in einer tief im (Unter)Bewusstsein verankerten Ablehnung der ,,anderen“ Gruppe aus. Der klassische Fall für ein Ressentiment ist der, oft mit Verschwörungstheorien und dem Kampf um innergesellschaftliche Dominanz verbundene, Antisemitismus (Decker und Brähler 2018, S. 185, 207). Diese Differenzen berücksichtigend, reflektieren allerdings sowohl Ressentiments als auch negativ gefärbte gruppenbezogene Vorurteile Ablehnungshaltungen gegenüber anderen sozialen Gruppen, verbunden mit deren Abwertung und einem Verständnis von „natürlicher“ Ungleichwertigkeit.

Bei der Betrachtung gruppenbezogenen Vorurteilen hat sich in Deutschland ein Konzept etabliert, dem in den meisten vorliegenden Studien das Gros der wissenschaftlich verwendeten Messinstrumente entnommen ist. Es handelt sich um den Ansatz der Gruppenbezogenen Menschenfeindlichkeit (Heitmeyer 2010; Zick und Klein 2014; Zick et al. 2016, 2019, S. 52-55). Das Konzept greift Aspekte der sozialpsychologischen Vorurteilsforschung, mit dem Schwerpunkt gruppenbezogener Vorurteile auf (Allport 1979; Tajfel 1982) und verdichtet diese zu einem generellen Befund. Alle diese Einstellungen sind den Autor*innen nach getragen von der Einschätzung der Ungleichwertigkeit anderer sozialer Gruppen der Gesellschaft der eigenen Gruppe gegenüber. ${ }^{6}$ Gruppenbezogene Vorurteile sind also stark untereinander verbunden und in unterschiedlichen Ausprägungen Ausdruck ein und derselben Neigung - der Gruppenbezogenen Menschenfeindlichkeit. Forschungstheoretisch wird ein gemeinsames Syndrom, vielleicht noch mit Varianten in der „Feindesgruppe“ angenommen. Es wird davon ausgegangen, dass Personen, die den entsprechenden Aussagen in repräsentativen Umfragen zustimmen, empfänglicher für Parolen sind, die auf Gruppenabgrenzung, Nationalismus und völkische Exklusivität zielen. In diesem Konzept wird die gestiegene Relevanz von Religion in diesem Sektor erkennbar. So wurden neben des Einbezuges von Antisemitismus (als Merkmal von Rechtsextremismus) auch abwertende Haltungen zu Islam und Muslimen in frühen Versionen der Skala Gruppenbezogene Menschenfeindlichkeit aufgenommen (siehe

\footnotetext{
${ }^{5}$ Ein Ressentiment wird oft tiefenpsychologisch analysiert. Gelegentlich werden Ressentiments stärker als Vorurteile an Emotionen und Affekte gebunden gesehen, während Vorurteile durchaus Erfahrungsbasiert sind.

${ }^{6}$ Bei Vorurteilen handelt es sich auch immer wieder um Kategorisierungen, welche stark mit Identität und Abgrenzung der eigenen Identität verbunden sind (Adida et al. 2016; Liedhegener 2016).
} 
Leibold und Kühnel 2003). Andere Religionsgemeinschaften stehen, aufgrund angenommener geringer Betroffenheit durch Vorurteile, nicht im Fokus der Forschung und werden nicht abgeprüft. Als Problem der Annahme eines Syndroms erweist sich der Verlust der inhaltlichen Reflexion der doch beachtlichen Differenzen in den Zustimmungsraten zu unterschiedlichen gruppenbezogenen Vorurteilen. So geraten diese vor dem Hintergrund des Gesamtkonzeptes etwas ins Hintertreffen. Gleichwohl hilft die Bündelung mit Blick auf eine gemeinsame Triefkraft für Vorurteile weiter.

Einen Bezugspunkt, den Heitmeyer $(2002,2018)$ ebenfalls aufgreift, ist die Wirkung der Einstellung des Autoritarismus. Er sieht diesen als einen von drei zentralen Faktoren für die Ausprägung Gruppenbezogener Menschenfeindlichkeit. Die Überlegungen zum Autoritarismus gehen auf Studien von Adorno (Adorno 1973, S. 315-328) zur autoritären Persönlichkeit zurück und identifizieren diese als eine auf Erziehungserfahrungen resultierende Persönlichkeitsstruktur. Die Abwertung des Anderen wird dabei zum Bedürfnis - und ist nur begrenzt Folge veränderter Lebensumstände. ${ }^{7}$ Spätere Studien erweiterten diese Vorstellungen in Richtung einer Mischung aus Persönlichkeitsmerkmalen und sozialer Einbettung (Decker und Türcke 2019; Pettigrew 1958). Entscheidend ist die Feststellung von Wirkungsbeziehungen. So wie sie Heitmeyer (2002) auf Gruppenbezogene Menschenfeindlichkeit feststellte, konnte Autoritarismus in jüngeren Studien immer wieder als Prädiktor für rechtsextreme Einstellungen identifiziert werden (exemplarisch Decker und Brähler 2018, S. 52-53, 174). Dabei wurde eine Prozesshaftigkeit herausgearbeitet und eine Dreiteilung in Konventionalismus, autoritäre Unterwerfung und autoritäre Aggression vorgenommen. Während Konventionalismus eine Orientierung am Konzept der Sicherheit und des Traditionalismus abbildet, beschreibt die autoritäre Unterwerfung den Wunsch nach Führung. Die autoritäre Aggression beschreibt das Bedürfnis, scheinbar schwächere oder abweichende Personen zu verfolgen oder ihre Verfolgung und Bestrafung zu fordern. Gerade letztere Komponente des Autoritarismus erweist sich als massiv Vorurteile fördernd. Auch Religion und Religiosität spielt hier eine Rolle. So betonte Adorno die funktionale Relevanz von Religion für Vorurteile: „Ethnozentriker halten Religion oft für eine praktische Hilfe zur seelischen Hygiene“ (Adorno 1973, S. 288). Speziell sieht Adorno Verbindungen zwischen parawissenschaftlichen Denkweisen des Religiösen und Vorurteilen. Religion kann allerdings auch als Medium der Verbreitung von Vorurteilen dienen (Adorno 1973, S. 426-450). Zudem zeigten sich Hinweise auf eine empirische Verbindung von religiösem Dogmatismus oder Fundamentalismus mit autoritären Einstellungen (Decker et al. 2018, S. 179-216). Dabei kamen neben Konkurrenzeffekten zwischen Religionsgemeinschaften auch Überschneidungen in konservativen und autoritären Vorstellungen der Gestaltung der Lebenswelt zu Wahlverwandtschaften zusammen. Entsprechend liegt es für die folgenden Analysen nahe, Bezüge zwischen einem dogmatischen Verständnis von Religion, Autoritarismus und Vorurteilen zu erwarten.

\footnotetext{
7 Dies führt auch dazu Antisemitismus in Abweichung von anderen Vorurteilen als Ressentiment mit einer umfassenden ideologischen Funktion einzuordnen (Adorno 1973, S. 110-115; Decker et al. 2018, S. 181-183; Ranc 2016).
} 


\subsection{Angst als (schlechter) Ratgeber? Integrated Threat Theory}

Dabei gehen wir davon aus, dass empfundene Bedrohungen in diesem Zusammenhang als Verstärker von Vorurteilen eine Rolle spielen. Hier stellt die Integrated Threat Theory den Ausgangspunkt unserer Überlegungen hinsichtlich der Wirkungen von Bedrohungsgefühlen dar. Dieser Ansatz ist der sozialpsychologischen Vorurteilsforschung zuzuordnen, an die auch Heitmeyer anschließt und der die Social Identity Theory (SIT) zugrunde liegt (Tajfel 1982). Letztere geht von einem grundsätzlichen Bedürfnis nach positiver Identität aus und das durch die Identifikation mit einer als positiv bewerteten Gruppe erreicht werden kann (Tajfel und Turner 1986, S. 10). ${ }^{8}$ Innerhalb der In-Group bildet sich eine gemeinsame Identität auf Grundlage von z.B. kulturellen Gemeinsamkeiten heraus. Sie gibt dem Einzelnen Selbstbewusstsein. Die In-Group profitiert durch eine Abwertung bzw. Unterordnung der Out-Group. Die Aufwertung der eigenen Gruppe führt, in Abgrenzung zu Anderen, zu einer Betonung insbesondere negativer Aspekte der Out-Group. Der zentrale Grund für die Mitglieder der Gruppe sich der In-Group anzuschließen ist also die Erhöhung des eigenen Selbstwertgefühls. Wichtig ist die emotionale Bindung des Gruppenmitglieds zu der jeweiligen In-Group. Gerade im Falle von geringer Gruppenzugehörigkeitsmobilität (social mobility) nimmt die emotionale Bedeutung zu und die Gruppenzugehörigkeit erlangt Bedeutung für die eigene Identität (Tajfel und Turner 1986, S. 11, 16).

Dieser Prozess birgt das Potenzial zur Ausbildung von Vorurteilen, ist es doch gerade die Abwertung anderer sozialer Gruppen, die den eigenen Selbstwert sichern helfen. Dadurch wird die Out-Group als ein von den eigenen Werten abweichendes Objekt betrachtet, welches ein Gefahrenpotenzial für die In-Group birgt. So entstehen im Abwertungsprozess Vorurteile gegenüber der anderen Gruppe, die sich festsetzen und Eigenständigkeit erlangen. Dies folgt einem klassischen Muster der Vorurteilsbildung über (1) Kategorisierung, (2) Stereotypisierung und (3) affektiver Aufladung (Zick et al. 2011, S. 32-35). Wird dieser Prozess mit Gefahrzuschreibungen verbunden, dann kommt es zu einer Bestärkung bzw. Verstärkung der Vorurteile, aufgrund einer Steigerung des emotionalen Investments (Jonas et al. 2014, S. 543-546; Hirschmann 1970). Diese Konstellation verursacht mit der Zeit die Entstehung von Ängsten und Bedrohungserleben. Während das Bild des ,gefährlichen Anderen“ immer stärker internalisiert wird, steigt der Stellenwert der Nähe zu der eigenen Kollektivgruppe. ${ }^{9}$ Unter Umständen entwickelt sich eine Dynamik, die zur

\footnotetext{
${ }^{8}$ Die SIT-Konzept geht davon aus, dass wir immer gleichzeitig einer Vielzahl von In-Groups angehören, die unsere Identität bestimmen. Wir konstituieren unsere Identität über die (Annahme) der Zugehörigkeit zu verschiedenen sozialen Gruppen. Allerdings kann uns diese Zugehörigkeit mehr oder weniger präsent bzw. wichtig sein. Dies gilt auch in Abhängigkeit von Situationen und Kontexten, welche die eigene Zugehörigkeit zu einer spezifischen In-Group salienter, bewusster und wichtiger erscheinen lassen, als die Zugehörigkeit zu anderen Gruppen. Die beschriebenen Abgrenzungsprozesse setzten in der Regel eine beachtliche Salienz der In-Group voraus.

9 Ein Verstärker für die Identifikation mit der Eigengruppe - und die soziale Abgrenzung von der Fremdgruppe - stellt das vom Individuum angenommene Ausmaß an von der Gruppe gewünschter Loyalität dar. Je loyaler eine Person gegenüber ihrer Gruppe glaube sein zu müssen, desto eher lehnt sie eine Fremdgruppe ab.
} 
Konstruktion weiterer gruppenbezogener Vorurteile führt und die Ablehnung der sozialen Gruppe zum Selbstzweck werden lässt.

Die solche Wirkungen von Bedrohungen fokussierende Integrated Threat Theory (ITT) (Stephan et al. 2000; Stephan und Stephan 1996) geht davon aus, dass diese Ängste und Zuschreibungen von Bedrohlichkeit eine Abgrenzung steigernde Wirkung besitzen (Jonas und Fritsche 2013). Die ITT unterscheidet zwischen symbolischen und realistischen Bedrohungen, mit denen sich die In-Group wie ihre Mitglieder konfrontiert sehen könnte. Symbolische Bedrohungen (symbolic threats) resultieren aus der wahrgenommenen Unterschiedlichkeit der In- und Out-Group. Die Sorge vor einer Verdrängung vermeintlich exklusiver eigener Werte durch die Out-Group ist eine Variante von symbolischer Bedrohung (Gonzalez et al. 2008, S. 669; Quillian 1995). Demgegenüber stehen die realistischen Bedrohungen (realistic threats), die sich anders als die symbolischen Bedrohungen auf physische, manifeste Bedrohungsgefühle zurückführen lassen. Dies kann die Angst vor terroristischen Anschlägen sein, aber auch eine Zunahme der Konkurrenz auf dem Arbeitsmarkt. Letztere thematisiert die eher ökonomisch angelegte Gruppenbedrohungstheorie (Blumer 1958; Blalock 1967). Die Bedrohungen werden der abgelehnten Referenzgruppe pauschal zugeschrieben. Aus diesen Bedrohungsgefühlen können Vorurteile entstehen, oder sie können diese zumindest massiv verstärken (Schlueter und Scheepers 2010). ${ }^{10}$ Die aus diesen Überlegungen ableitbare Annahme ist, dass eine starke Wahrnehmung einer Bedrohung durch eine religiöse Gemeinschaft und ihre Mitglieder deren Abwertung bestärkt (auch Uenal 2016). Dabei ist es wichtig diesen Prozess nicht als eine „,nur durch das Gefühl von Bedrohung“ erzeugte Abwertung und Ablehnung zu verstehen, die durchaus akzeptabel scheint, sondern als einen Bestandteil der Verstärkung und Verbreitung von Vorurteilen in einer Gesellschaft, in der bereits Ansätze von Vorurteilen und ggf. Ressentiments vorliegen. So schließt eine Identifikation von Beziehungen zwischen Bedrohungsgefühlen und Vorurteilen keineswegs die Existenz von Rassismus und Ressentiments aus. ${ }^{11}$

\subsection{Der Gegenpart zum Abbau von Ängsten - die Kontakthypothese}

Immer wieder geht aus den Daten verschiedener Studien hervor, dass Ressentiments gegenüber Muslim*innen besonders an Orten verbreitet sind, in denen der Anteil der muslimischen Bevölkerung sehr gering ausfällt (Pickel und Öztürk 2018). Wie lässt sich dies in das Konzept der Integrated Threat Theory einbetten? Hier kann

\footnotetext{
10 Gleichzeitig haben bereits Stephan und Renfro (2002) auf die wechselseitige Beeinflussung von Vorurteilen und Bedrohungswahrnehmungen hingewiesen. So sind entsprechende Kausalannahmen kontextuell herzuleiten oder zumindest vorsichtig zu verwenden.

11 Im vorliegenden Aufsatz wird auf Ansätze aus der Vorurteilsforschung zurückgegriffen. Gleichzeitig ist es möglich auch Theorien die sich kritisch zu Rassismus verhalten zu berücksichtigen. Diese gehen von Abwertungsannahmen und Selbstaufwertungsmechanismen aus, die teilweise gut mit den verwendeten sozialpsychologischen Erklärungen übereinstimmen. Angelegt an Überlegungen von Albert Memmi (1987) wollen wir den Begriff des Rassismus hier eng fassen und ihn entsprechend für unsere Betrachtung von Vorurteilen gegenüber Religionsgemeinschaften vorerst zurückstellen, selbst wenn er im Zuge von Ethnisierungsprozessen durchaus ebenfalls seine Rechtfertigung besitzt (siehe z. B. Cakir 2014).
} 
die sogenannte Kontakthypothese, eine weitere sozialpsychologische Theorie, weiterhelfen (Allport 1979; Allport und Ross 1967; Pettigrew 1998). Sie legt nahe, dass der konkrete Kontakt zur jeweiligen Out-Group die Bedrohungsgefühle - und damit die Vorurteile - verringern kann. Die Idee ist, dass persönliche Kontakte positive Erfahrungen mit sich bringen, oder zumindest Vorstellungen von Normalität erzeugen. Dieser mit Erfahrung verbundene Kontakt wirkt Verschwörungstheorien oder nicht durch Kontakterfahrungen abgesicherten Vorurteilen entgegen. Bereits Allport nahm allerdings Einschränkungen vor, die später wiederholt aufgenommen wurden: Zum beurteilte er die Annahme einer universalisierten positiven Wirkung von Kontakten als zu oberflächlich, zum anderen verweis er auf die große Variation von Kontakten und Kontaktkontexten (Allport 1979). Speziell die Berücksichtigung von Statushierarchien in den Kontakten, Kooperationsbereitschaft und gemeinsame Ziele wurden als förderlich für den Kontakterfolg angesehen (Jonas et al. 2014, S. 548-550; Sherif et al. 1961; Brown und Abrams 1986). So bedenkenswert diese kritischen Anmerkungen sind, konnte die empirische Forschung doch wiederholt die Effektivität von Kontakten belegen. So erbrachte eine breite Metaanalyse von Pettigrew und Troop (2006) den Hinweis auf eine überwiegende Bestätigung der Kontakthypothese. Auch die Naivitätsannahme musste eingeschränkt werden, verweis doch Allport (1979) selbst frühzeitig auf die Bedeutung von Differenzen im Status von Kontaktgruppen hin oder betonte die hohe Relevanz des normativen Klimas im Umfeld (Pettigrew 1998). Positive Framings und Voraussetzungen unterstützen die positive Wirkung von Kontakten, wie auch Varianten der Kontakthypothese gezielte Interventionsmöglichkeiten gegenüber Vorurteilen bereitstellen (Hewstone und Swart 2011). Gleichzeitig kann die Hypothese einer positiven Wirkung von Kontakten auf den Abbau von Vorurteilen aufrecht erhalten bleiben.

Interessant ist zudem für unsere Zwecke die Erweiterung der Kontakthypothese durch para-soziale Kontakte (Horton und Wohl 1956). Wenn keine Möglichkeit des direkten sozialen Kontakts besteht, ist der parasoziale Kontakt die nächstgelegene Informationsquelle. Für ihn ist kein direkter Kontakt notwendig. Parasozialer Kontakt kann etwa in Form von medialer Berichterstattung stattfinden und das Meinungsbild innerhalb der Bevölkerung in Bezug auf eine bestimmte Bevölkerungsgruppe prägen. Ein gutes Beispiel für solche Wirkungen stellt die Einschätzung der muslimischen Bevölkerung dar. So fällt z.B. die mediale Rezeption für die Gruppe der Muslim*innen deutlich negativ aus. Yasin Baş hält in seiner Presseschau für 2018 etwa die Themen Flüchtlingskrise, IS, Terroranschläge, steigender Antisemitismus in Deutschland, Probleme bei Abschiebungen, Islamistisches Gefahrenpotential in Deutschland und Angelegenheiten des Verfassungsschutzes als einige relevante Bereiche für die Berichterstattung (Baş 2019, S. 11-45). Zu ähnlichen Befunden kommen Hafez und Schmidt (2015). Die mediale Rezeption zur muslimischen Bevölkerungsgruppe liefert eine wichtige Voraussetzungen für die genannten Formen der Bedrohungsgefühle - symbolische wie realistische Bedrohungen. Während realistische Bedrohungsgefühle ein Produkt para-sozialer Kontakte mit Islamisten und radikalen Gruppierungen im Islam darstellen, referieren symbolische Kontakte auf eine undefinierbare, kulturelle Bedrohung. Diese kann durch Debatten über Kopftuch- oder Burkaverbote intensiviert werden (z.B. Carol et al. 2015) und findet 
teilweise Übergänge zu einem Ressentiment oder rassistischen Positionen im Rahmen diskursiver Ethnisierung (Shooman 2014).

Es zeigen sich allerdings Unterschiede zwischen einer breiteren, durchaus auch mit Vorurteilen behafteten Sichtweise und einem ethnisch geprägten Rassismus. So wie Rassismus und rassistische Vorstellungen vorherrschen, scheinen sie doch seltener verbreitet als die Bedrohungsgefühle. Nicht, dass aus den Vorurteilen heraus rassistische Einstellungen werden können oder diese im Rahmen der Ethnisierung belebt werden. Insgesamt können allerdings Effekte von Kontakten, positive bei direkten und persönlichen Kontakten zu Mitgliedern einer Religionsgemeinschaft, negative bei para-sozialen Kontakten oder keinen Kontakten erwartet werden. Die Vermutung ist, dass Kontakte bestehende Bedrohungsgefühle konterkarieren und zu ihrem Abbau beitragen, liegen nicht sowieso bereits grundsätzlichere Ressentiments oder gar Rassismus vor.

\section{Methodik: Die Messung gruppenbezogener Vorurteile im Berlin- Monitor}

In aktuellen gesellschaftlichen Diskussionen spielt die Haltung zu anderen sozialen Gruppen eine große Rolle. So wird in den letzten Jahren öffentlich eine Zunahme von Rechtspopulismus und Rechtsextremismus, wie von antimuslimischem Rassismus und Antisemitismus diskutiert (Pickel und Pickel 2019; RIAS 2019). Diese Diskussionen können allerdings oft auf keine klaren empirischen Belegstrukturen zurückzugreifen, da sich das Phänomen das Populismus weitgehend durch eine unklare Messstruktur auszeichnet (statt vieler Castanho Silva et al. 2019). Deutlich besser zu fassen (wenn auch in Teilen mit Einschränkungen) sind rechtsextreme Einstellungen, Aussagen sozialer Abwertung, Ungleichheitsvorstellungen, Rassismus, gruppenbezogene Vorurteile und Ressentiments. Diesen Einstellungen wohnt ein bedrohliches Potential für die liberale Demokratie inne (Decker et al. 2016), stellen sie doch ein Einfallstor für Rechtsextremismus und Nichtdemokraten in gesellschaftliche Debatten und Bezüge dar. Soziale Distanzen und Abneigungen, wie Stereotypen und Vorurteile werden zu Grundvorstellungen vieler Menschen und führen zu antidemokratischen und antipluralistischen Positionen. Dabei reichen Vorurteile und Abwertung weit über Rassist*innen und Rechtsextremist*innen hinaus, teilweise bis in gesellschaftlichen Schichten, welche man als politische Mitte bezeichnen könnte (siehe Decker et al. 2016; Rippl und Seipel 2018; Zick et al. 2019). Um sie empirisch fassen zu können wurden in den letzten Jahrzehnten verschiedene Erhebungsinstrumente konzipiert. Sie alle basieren auf dem System von vorgegebenen Aussagen, welche in ihrer Auswertung Auskunft über Vorurteile und soziale Ungleichwertigkeitswahrnehmungen geben sollen. ${ }^{12}$ Dabei muss man sich klar sein, dass man allein auf der Ebene dieser Operationalisierung nicht zwischen Vorurteilen und Ressentiments unterscheiden kann, fehlt doch die Verbindung zum Grund der Ablehnung und Abwertung. In der Einordnung hilft dementsprechend nur eine

\footnotetext{
12 Selbst wenn die entsprechenden Aussagen teilweise verstörend oder unpassend wirken können, leisten sie einen guten Einblick in entsprechende Vorurteile.
} 
aus der Theorie begründete Entscheidung. Auf dieser Basis entscheiden wir uns Antisemitismus als Ressentiment und Ablehnungshaltungen gegenüber dem Islam vorerst als gruppenbezogene Vorurteile einzuordnen. Gleichwohl wäre eine andere Einordnung der zuletzt genannten Haltung, z. B. als antimuslimisches Ressentiment oder antimuslimischer Rassismus möglich. Da es uns aber im Kern um inhaltliche Erkenntnisse geht, lassen wir weitere definitorische Diskussionen hier offen und legen - quasi etwas autoritär - eine möglichst gut empirisch umsetzbare Definitorik fest.

Unterschiedliche der potentiell von Vorurteilen betroffenen Gruppen werden im bereits angesprochenen Konzept der Gruppenbezogenen Menschenfeindlichkeit (GMF) erfasst (Heitmeyer 2010; Zick et al. 2019). Die Kernannahme im Konzept der Gruppenbezogenen Menschenfeindlichkeit ist, dass es sich bei diesen verschiedenen Einzelvorurteilen um ein miteinander verbundenes Syndrom und ein vernetztes Phänomen handelt. Dieses Syndrom wird nach Ansicht der Autoren des Konzeptes getragen von generellen Vorstellungen der Ungleichwertigkeit verschie-

Tab. 1 Gruppenbezogene Vorurteile und ihre Indikatoren (Konzeptionelle Zuordnung)

Gruppenbezogene Vorurteile (und Ressentiments)

Feindlichkeit gegen Homo- bzw. Bisexuelle (Homophobie)

Homosexualität ist etwas völlig normales

Homosexualität ist eine Krankheit, die geheilt werden kann

Es sind zwingend Maßnahmen gegen die Diskriminierung von Lesben, Schwulen und Bisexuellen nötig

Feindlichkeit gegen Transsexuelle/Transgender (Transphobie)

Transsexualität ist eine Gefahr für die gesellschaftliche Ordnung

Anti-Feminismus

Überzogene Forderungen der Gleichberechtigung richten sich gegen die Natur von Frauen und Männern

Feindlichkeit gegen Arbeitslose (Soziale Deklassierung)

Die meisten Hartz IV-Empfänger machen sich auf Kosten anderer ein schönes Leben

Feindlichkeit gegenüber Geflüchteten

Flüchtlinge müssen in Deutschland immer willkommen sein

Die Zuwanderung von Asylbewerbern in unser Land muss unterbunden werden

Antiziganismus

Sinti und Roma sollten aus den Innenstädten verbannt werden

Feindlichkeit gegen Schwarze (Anti-Schwarzer Rassismus)

Es gibt eine natürliche Hierarchie zwischen Schwarzen und Weißen Menschen

Schwarze Menschen können in Deutschland nicht zurechtkommen

Feindlichkeit gegenüber Muslim*innen (bzw. antimuslimische Ressentiments)

Die Anzahl der Muslime in Deutschland ist zu hoch

Muslimen sollte die Zuwanderung nach Deutschland untersagt werden

Antisemitische Ressentiments

Auch heute noch ist der Einfluss der Juden zu groß

Reparationsforderungen an Deutschland nutzen oft gar nicht den Opfern, sondern einer Holocaust-Industrie von findigen Anwälten

Israels Politik in Palästina ist genauso schlimm, wie die Politik der Nazis im zweiten Weltkrieg

Anmerkungen: Formulierungen entsprechen dem exakten Wortlaut der entsprechenden Items bei der Berlin-Monitor Umfrage 2019; $N=2005$ Berliner*Innen 
dener Menschen in einer Gesellschaft. Auch jenseits einer zusammenfassenden Verwendung der erhobenen Vorurteile sind die Erhebungen einzelner Referenzgruppen von Abwertung für eine Kartographie gruppenbezogener Vorurteile hilfreich, werden doch Abgrenzungsbedürfnis und Betroffenengruppen erkennbar. So belegen Ergebnisse mit dem Erhebungsinstrument der GMF immer wieder beachtliche Differenzen im Umfang der Vorurteile, je nach jeweiliger Referenzgruppe. Teile dieses Instrumentariums wurden auch im Berlin-Monitor 2019 eingesetzt. Da einige Fragestellungen der klassischen GMF-Skala aufgrund des Voranschreitens der Debatten in die Diskussion geraten sind und zudem einige Referenzgruppen gruppenbezogener Vorurteile nicht enthalten sind, wurde die Erhebung der Vorurteile an einigen Stellen ausgeweitet und Fragestellungen variiert (siehe Tab. 1). Dafür muss das Risiko von Abweichungen gegenüber anderen, bundesweiten Studien, aufgrund unterschiedlicher vorgegebener Statements, in Kauf genommen werden. Im Berlin-Monitor versuchen wir durch eine Mischung aus negativ und positiv ausgerichteten Aussagen der üblichen, dominanten Ausrichtung auf abwertende Urteile zu begegnen. Auf diese Weise wird man den methodischen Bedürfnissen von Skalenkonstruktion, nämlich Aussagen mit Ausrichtung auf die beiden Pole einer Dimension, besser gerecht als teilweise in bisherigen Erhebungen. In der folgenden Tab. 1 sind die verschiedenen erhobenen Konstrukte aufgeführt.

Als Ergänzung zu teilweise aus anderen Studien übernommenen Aussagen im Bereich Antisemitismus und der Muslimfeindlichkeit, wurde die Abwertung Schwarzer Menschen, die Abwertung Transsexueller/Transgender und Bisexueller, aber auch die zuletzt immer häufiger in den Blick kommende Beobachtung des gegen Gleichstellung und Frauenrechte gerichteten ,Anti-Feminismus“ im Berlin-Monitor berücksichtigt. ${ }^{13}$ Ebenfalls einbezogen wurden Erhebungsinstrumente für Homophobie, Transphobie, Antiziganismus, Feindlichkeit gegenüber Geflüchteten, antimuslimische Ressentiments und Antisemitismus. Für den Antisemitismus verwenden wir eine dreigliedrige Messung, in der neben dem (klassischen) primären Antisemitismus, sekundärer Antisemitismus (inklusive Schuldabwehrantisemitismus) sowie israelbezogener Antisemitismus enthalten sind. Während die Abfrage zu Antisemitismus aus Vergleichsgründen anhand einer Skala mit fünf Antwortmöglichkeiten durchgeführt wurde, erfolgte die Messung der Vorurteile über eine 4-Punkt-Skala (ebenfalls untereinander vergleichbar).

\section{Gruppenbezogene Vorurteile in Berlin - Unterschiedliche Grade}

\subsection{Gruppenbezogene Vorurteile in Berlin in der Übersicht}

Gehen wir in die Daten und werfen einen ersten Blick auf die Verteilung der Vorurteile und Ressentiments. Die Berliner*innen stehen den im Berlin-Monitor abgefragten Gruppen mehrheitlich positiv gegenüber. Selbst die heftig diskutierten

\footnotetext{
13 In ersten Veröffentlichungen haben wir den Bereich des Anti-Feminismus als Antigenderismus behandelt. Bei längerer Überlegung erscheint uns die neue Bezeichnung dem Gegenstand besser angemessen (siehe Pickel et al. 2019).
} 
Fragen der Zuwanderung - speziell der muslimischen Zuwanderung - führen zu keiner mehrheitlichen Ablehnung unter den Berliner*innen. Vorurteile sind also im pluralistischen Berlin - wie ein wenig erwartet - ein Minderheitenphänomen. Doch selbst wenn sich keiner sozialen Gruppe gegenüber eine Mehrheit an ablehnenden Haltungen findet, existiert eine ansehnliche Bandbreite sozialer Abwertung und von sozialen Ungleichwertigkeitsvorstellungen. Die Einstellungen gegenüber den beiden religiösen Gruppen - Muslim*innen und Jüd*innen - fallen unterschiedlich aus. ${ }^{14}$ Während der Antwortvorgabe für antimuslimische Vorurteile relational mit am häufigsten, wenn auch nicht mehrheitlich, zugestimmt wird, bewegen sich antisemitische Äußerungen eher im Mittelfeld der abgefragten Vorurteile. ${ }^{15}$ Nun ist es aufgrund der Ungleichheit der Frageformulierungen schwer, bis nur begrenzt zulässig, direkte Zustimmungsvergleiche zwischen den Gruppenabwertungen vorzunehmen. Gleichwohl kann man über Plausibilitätsüberlegungen doch aus den Relationen vorsichtige Schlussfolgerungen ableiten.

Richten wir unser Augenmerk auf die religiösen Gruppen. Beginnen wir mit den Muslim*innen, da diese nach Adida et al. (2016) die größten Schwierigkeiten besitzen sollen in christlichen Gesellschaften Fuß zu fassen. Selbst wenn wir Berlin von der Zugehörigkeit als mehrheitlich konfessionslos oder säkular einstufen können, besteht doch ein christlicher Kulturhintergrund. Nur 13\% der Berliner*innen stimmen einem generellen Zuwanderungsstopp für Muslim*innen zu, obwohl immerhin $29 \%$ der Berliner*innen die Zahl der Muslim*innen in Deutschland als zu hoch empfindet. ${ }^{16}$ Dieser Wert (Zuwanderungsstopp) liegt unter dem Bundesschnitt von $18 \%$ (Zick et al. 2019, S. 72-73). Damit findet sich eine Zwischengruppe von Berliner*innen, die zwar eine gewisse kulturelle „Überfremdung“ durch Muslim*innen empfinden, aber daraus nicht die Forderung eines Zuwanderungsstopps ableiten. Zwischen der Sicht auf Muslim*innen und Geflüchtete besteht eine enge Verbindung (Pickel und Pickel 2018, 2019, S. 297). Die statistischen Überschneidungen im Antwortverhalten gegenüber Muslim*innen und Geflüchteten sind beträchtlich (in der Regel Korrelationen um $r>0,50$ ). Geflüchtete werden von vielen der befragten Berliner*innen gleichzeitig als Muslim*innen eingestuft (Pickel und Pickel 2019, S. 295-298) (Abb. 1).

Entsprechend ist die Ablehnung von Geflüchteten auf ähnlichem Zustimmungsniveau, wie das Antwortverhalten auf die Frage, nach einem Zuwanderungsstopp für Geflüchtete, den $17 \%$ der Berliner*innen befürworten, belegt. Im Berlin-Monitor haben wir zudem mit einer positiven Frageformulierung die Haltung gegenüber Geflüchteten ermittelt. Das Ergebnis ist eindeutig: Drei Viertel der Berliner*innen finden, dass Flüchtlinge in Deutschland immer willkommen sein müssten. Von einer

\footnotetext{
${ }^{14}$ Wobei hier die Kommunikationslatenz, die Schuldverdrängung und die Umwegkommunikation antisemitischer Einstellungen bedacht werden muss (Beyer und Krumpal 2010).

15 Die aufgezeigten Ergebnisse decken nicht die Betroffenenperspektive ab und sollten keinesfalls, als eine mehr- oder weniger-Betroffenheit für Einzelne interpretiert werden. Die Vorurteilsmessung gibt Auskunft darüber, inwiefern die Abwertung bestimmter Gruppen besonders weit verbreitet ist.

16 Nicht gefragt wurde das Item „Fühle mich manchmal durch die Muslime als Fremder im eigenen Land“. Diese Aussage ist in ihrer Zielrichtung unklar du sehr leicht zustimmungsfähig, was Zustimmungsraten zwischen 36\% (Zick et al. 2019, S. 71) und 56\% (Decker und Brähler 2018, S. 102; mit anderem Umfragedesign) belegen.
} 
Homosexualität ist etwas völlig normales.

Homosexualität ist eine Krankheit, die geheilt werden kann.

Es sind zwingend Maßnahmen gegen die Diskriminierung von Lesben, Schwulen und Bisexuellen nötig.

Transsexualität ist eine Gefahr für die gesellschaftliche Ordnung.

Überzogene Forderungen der Gleichberechtigung richten sich gegen die Natur von Frauen und Männern.

Die meisten Hartz IV-Empfänger machen sich auf Kosten anderer ein schönes Leben.

Schwarze Menschen können in Deutschland nicht zurechtkommen.

Es gibt eine natürliche Hierarchie zwischen schwarzen und weißen Menschen.

Sinti und Roma sollten aus den Innenstädten verbannt werden.

Die Zuwanderung von Asylbewerbern in unser Land muss unterbunden werden.

Flüchtlinge müssen in Deutschland immer willkommen sein.

Die Anzahl der Muslime in Deutschland ist zu hoch.

Muslimen sollte die Zuwanderung nach Deutschland untersagt werden.

Auch heute noch ist der Einfluss der Juden zu groß.

Reparationsforderungen an Deutschland nutzen oft gar nicht den Opfern, sondern einer Holocaust-Industrie von findigen... Israels Politik in Palästina ist genauso schlimm wie die Politik der Nazis im zweiten Weltkrieg.

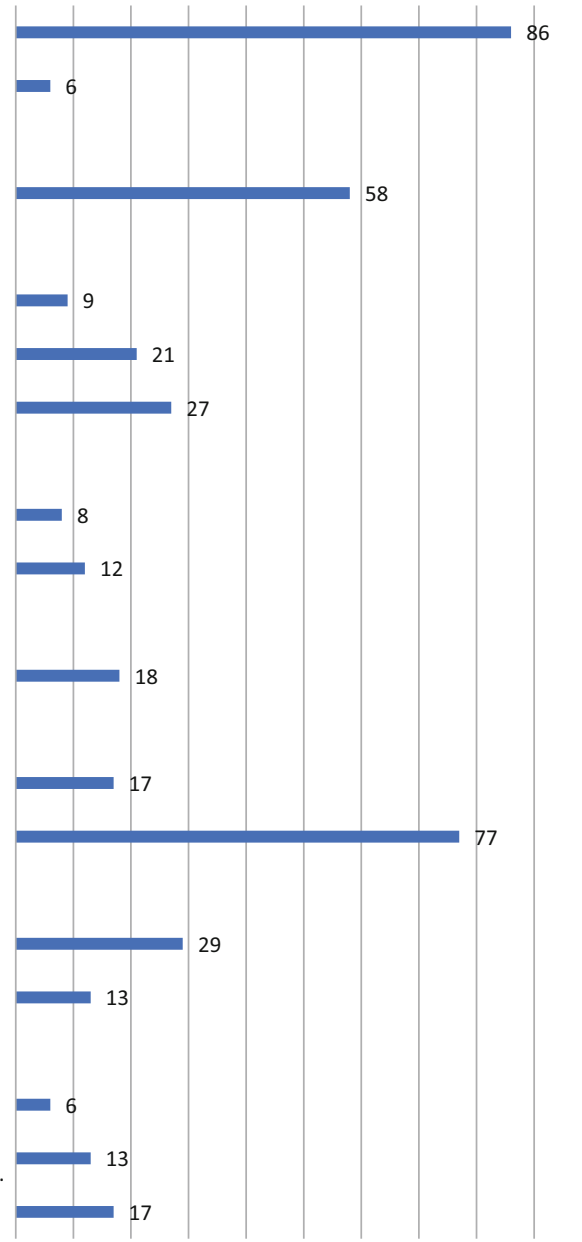

Abb. 1 Gruppenbezogene Vorurteile in Berlin im Überblick (in \%). Quelle: Eigene Darstellung auf Grundlage der Ergebnisse der Berlin-Monitor Umfrage; $N=2005$ Berliner*Innen; Ausgewiesen sind gültige Prozentwerte der Zustimmung zu den jeweiligen Aussagen (stimme stark zu, stimme eher zu) bei vier Antwortvorgaben; Daten repräsentativ gewichtet

weit verbreiteten oder gar generellen Ablehnung von Fluchtzuwanderung kann zumindest in Berlin - nicht die Rede sein. Ähnlich Verhältnisse zeigt das Antwortverhalten in Richtung Muslim*innen: Die Berliner Gesellschaft teilt sich in eine drei Viertel-Gruppe und eine ein Viertel-Gruppe. Ein Viertel der Berliner*innen sieht eine Offenheit für Geflüchtete und Muslim*innen nicht als angebracht und befürwortet einen Einreisestopp. ${ }^{17}$ Umgekehrt finden drei von vier Berliner*innen,

17 Die verwendete Neukonstruktion ergibt ein besseres Bild über die Haltung zu Geflüchteten gegenüber den bislang eingesetzten Fragen danach, ob ,der Staat bei der Prüfung von Asylanträgen großzügig sein sollte“ oder inwieweit „Asylbewerber in ihrem Heimatland gar nicht verfolgt werden“ (Zick et al. 
dass Flüchtlinge immer in Deutschland willkommen sein müssten, was auf eine weit verbreitete interkulturelle Toleranz in Berlin hinweist.

Wie in bundesweiten Umfragen sind antisemitische Einstellungen in Berlin relativ wenig verbreitet. Dabei ist nicht zu bestimmen, wie hoch die soziale Erwünschtheit bei entsprechenden Fragen ist. So finden sich aufgrund der prekären deutschen Geschichte Zustimmungsblockaden und Effekte sozialer Erwünschtheit, speziell, wenn es um den primären Antisemitismus geht. Dieser ist im Berlin-Monitor auf dem niedrigem Bundesniveau (Decker und Brähler 2018; Zick et al. 2019). Allerdings finden sich Unterschiede zwischen dem primären Antisemitismus, dem sekundären Antisemitismus mit einem Schuldverdrängungskomplex und einem israelbezogenen Antisemitismus (Frinte und Wammelsberger 2008; Pickel et al. 2019, S. 56; auch Lipstadt 2019). Die Werte des sekundären Antisemitismus, die eine Art Schuldabwehrantisemitismus beschreiben (Schönbach 1961, S. 80-81), fallen höher aus als die Zustimmung zum primären Antisemitismus. Der israelbezogene Antisemitismus liegt noch etwas höher. ${ }^{18}$ Wenn auch die große Mehrheit der Berliner*innen keine Antisemit*innen sind, finden sich doch größere Spurenelemente einer Umwegkommunikation des Antisemitismus in Berlin. So werden, aufgrund der sozialen (Un)Erwünschtheit von Antisemitismus in Deutschland, eigentlich bestehende antisemitische Einstellungen anders, über den Umweg einer gegen Israel gerichteten Kritik, Abwertung und Ablehnung oder unter Verweis auf eine übertriebene Konzentration auf den Nationalsozialismus in der Behandlung der deutschen Historie zum Ausdruck gebracht.

Wie sieht es mit anderen Vorurteilen aus. Generell fallen die Ergebnisse im Vergleich zu bundesweiten Studien eher moderater aus. Dies zeigt sich zum Beispiel bei der Abwertung von Sinti und Roma (Zick et al. 2019, S. 72-73). Auf niedrigem Niveau, nimmt man bundesweite Ergebnisse als Referenzpunkt, liegt die Abwertung von Sinte*zze und Rom*nja (17\%). Dieser Wert ist im Bundesvergleich als eher moderat einzustufen. Auffällig ist die relativ hohe Bereitschaft zur sozialen Deklassierung, festgemacht an der Abwertung von Hartz-V-Empfängern. Soziale Disparitäten werden in einer stark auf Leistung ausgerichteten Gesellschaft von nicht wenigen Menschen als gerechter Ausdruck des individuellen Einsatzes gesehen - und sozial Deprivierte dann als selbst an ihrem Unglück schuld. Die stärksten Vorurteile unter den Berliner*innen existieren unter den abgefragten Referenzgruppen gegenüber Langzeitarbeitslosen. $27 \%$ gehen davon aus, dass sie sich auf Kosten der Allgemeinheit ein „schönes Leben“ machen. Diese starke Betroffenheit von sozialer Abwertung nehmen sie auch in bundesweiten Umfragen (bei einem variierten Befragungszugriff) ein, wo ungefähr $50 \%$ der Deutschen davon ausgehen, dass Langzeitarbeitslose gar keinen Arbeitsplatz suchen (Zick et al. 2019, S. 74-75).

2019, S. 72-73). Dies zeigt auch die Notwendigkeit von neuen Messkonstruktionen für die Erfassung von gruppenbezogenen Vorurteilen. Um diese, und auch Haltungen zu Geschlechtergleichheit sowie Homo-/ Bisexualität angemessener fassen zu können, wurde in der Befragung zudem versucht neben Negativaussagen auch Positivaussagen zu verwenden.

18 Dabei erweist sich gerade die Bestimmung eines israelbezogenen Antisemitismus in Relation zu ,,berechtigter" Israelkritik immer als schwierige Aufgabe. Gleichwohl erscheint uns das hier verwendete Item einen starken antisemitischen Charakter aufzuweisen und korrespondiert auch statistisch mit den anderen Ausdrucksformen des Antisemitismus. 
Dem klassischen Rassismus am nächsten kommt die Haltung gegenüber People of Colour (POC) oder Schwarzen Menschen. Nur $8 \%$ der Befragten nehmen an, dass sich Schwarze Menschen in Deutschland nicht zurechtfinden können. Mehr zu denken gibt eine fundamentale Ungleichwertigkeit abbildende Aussage, welche eine (natürlichen) Hierarchie zwischen Weißen und Schwarzen Menschen sieht. Sie wird immerhin von knapp $12 \%$ der Berliner*innen bejaht. Selbst wenn Abwertungen von Schwarzen Menschen kein Mehrheitsphänomen sind, existieren sie doch auch im multikulturellen Berlin.

Neben diesen sozialen Gruppen rücken in aktuellen Diskussionen immer wieder Fragen der sexuellen Orientierung, Geschlechtsidentität und Geschlechtergleichheit in den Fokus. Im Berlin-Monitor bestätigt sich unter den Berliner*innen das Bild einer weitgehenden Akzeptanz einer selbstbestimmten Ausübung von Sexualität. 86\% der Berliner*innen sehen Homosexualität als etwas vollkommen normales an und $83 \%$ widersprechen in vollem Umfang der Aussage, dass Homosexualität heilbar wäre. Gerade die zweite Aussage beschreibt ein problematisches Verständnis von Homosexualität, dass speziell in Kreisen dogmatischer Christ*innen immer noch Relevanz besitzt. ${ }^{19}$ So wird Homosexualität mit der Zustimmung zu einer solchen Formulierung - immerhin noch bei 5-6\% der Berliner*innen - als Krankheit eingeordnet. Ähnlich verhält es sich beim Antwortverhalten hinsichtlich Transsexualität. Wieder sind es ca. 9\%, welche Transsexualität (sogar) als Gefahr für die gesellschaftliche Ordnung einschätzen. Hier handelt es sich um eine explizite Ablehnung einer sozialen Gruppe, welche die eigenen gesellschaftlichen Normen und ihre Gültigkeit in Frage zu stellen droht. Fasst man die Ergebnisse zusammen, dann wird von einer, wenn auch kleinen, Gruppe von Berliner*innen die Verbreiterung binärer Geschlechtsidentitäten in Frage gestellt oder abgelehnt. Insgesamt sind in Berlin die vielfältigen Lebensformen breitflächig akzeptiert. Nur eine kleine Minderheit steht ihnen nicht aufgeschlossen gegenüber und lehnt sie ab. Allerdings sollte man hinsichtlich einer zu weit reichenden positiven Interpretation der Akzeptanz pluraler Lebensformen und Geschlechtsidentitäten Vorsicht walten lassen. So reduziert sich die Zahl der toleranten Personen, wenn man die Vorgabe variiert. So fällt die Befürwortung von Maßnahmen zur Bekämpfung der Diskriminierung von Lesben, Schwulen und Bisexuellen auf die mittlere Größe von $57 \%$ der Berliner*innen. Dies ist an sich kein schlechter Wert, zeigt aber eine Differenz zwischen dem Toleranzmodell einer passiven Duldung und dem Toleranzmodell einer aktiven Akzeptanz (Forst 2003) - oder einer auf Wertschätzung basierenden Toleranz (Klein 2014, S. 59). Da ist es beachtlich, dass nur ein Fünftel der Berliner*innen sieht, dass sich „überzogene Forderungen der Gleichberechtigung“ gegen die Natur von Männern und Frauen richten. Gerade mit dem Präfix , überzogene“ wäre eine höhere Ablehnung ohne weiteres möglich gewesen. Nichtsdestotrotz sollte man die Einschätzung einer, hinsichtlich einer Ausweitung von Gleichberechtigung skeptischen Haltung eines doch sichtbaren Teils der Berliner*innen, nicht ignorieren. ${ }^{20}$

\footnotetext{
19 Dies wird auch daran deutlich, dass das Gesundheitsministerium der Bundesrepublik Deutschland sich genötigt sah, entsprechende Therapien explizit zu verbieten.

20 Ein interessanter Befund ist, dass die Haltungen gegenüber Gleichstellungsfragen zwischen Männern und Frauen nicht variieren. Entgegen der vielleicht existierenden Annahme, es wären gerade Männer,
} 
Eine solch aufmerksame Deutung ist für alle der betrachteten gruppenbezogenen Vorurteile angebracht. Insgesamt sind die Zustimmungsgrade zu den einzelnen gruppenbezogenen Vorurteilen auf deutliche Minderheiten der Berliner*innen begrenzt. Allerdings befürworten immerhin $45 \%$ der Berliner*innen zumindest ein gruppenbezogenes Vorurteil. Selbst wenn eine solche additive Messung, noch dazu bei relativ vielen Antwortvorgaben, wie im Berlin-Monitor vorgelegt, methodische Schwierigkeiten beinhaltet, zeigt dies eine fluide Verbreitung von (teils unterschiedlichen) Ungleichwertigkeitsvorstellungen in einem beachtlichen Teil der Berliner Bevölkerung. Selbst wenn die Mehrheit der Berliner*innen vorurteilsfrei ist und $15 \%$ nur eine Antwort unterstützen.

\subsection{Gruppenbezogene Menschenfeindlichkeit oder unterschiedliche Dimensionen}

Vorurteile können untereinander stark verzahnt sein. So ist die Wahrscheinlichkeit, dass jemand Muslim*innen ablehnt und eine kritische Haltung zu Genderfragen besitzt hoch, wenn der Zusammenhang auch nicht natürlich gegeben und zwingend ist. Eine solche Verzahnung entspricht den Annahmen im Konzept der gruppenbezogenen Menschenfeindlichkeit (Zick et al. 2019, S. 76-77), verweist aber auch darüber hinaus. So ist es genauso plausibel das Zusammenfallen verschiedener Ablehnungshaltungen als Effekt einer autoritären Aggression (Decker und Brähler 2018, S. 172) $\mathrm{zu}$ interpretieren, quasi mit einer in der Sozialisation internalisierten persönlichen Disposition als gemeinsamen Ausgangspunkt für Vorurteile und Ressentiments.

Korrelationsanalysen zwischen den verschiedenen Indikatoren der sozialen Abwertung erbringen dann auch starke empirische Beziehungen zwischen allen Ungleichwertigkeitsvorstellungen. Allerdings besitzen diese bei weitem keine $100 \%$ ige Beziehungsstärke (Tab. 2). Bestimmte Abwertungen sind enger miteinander ver-

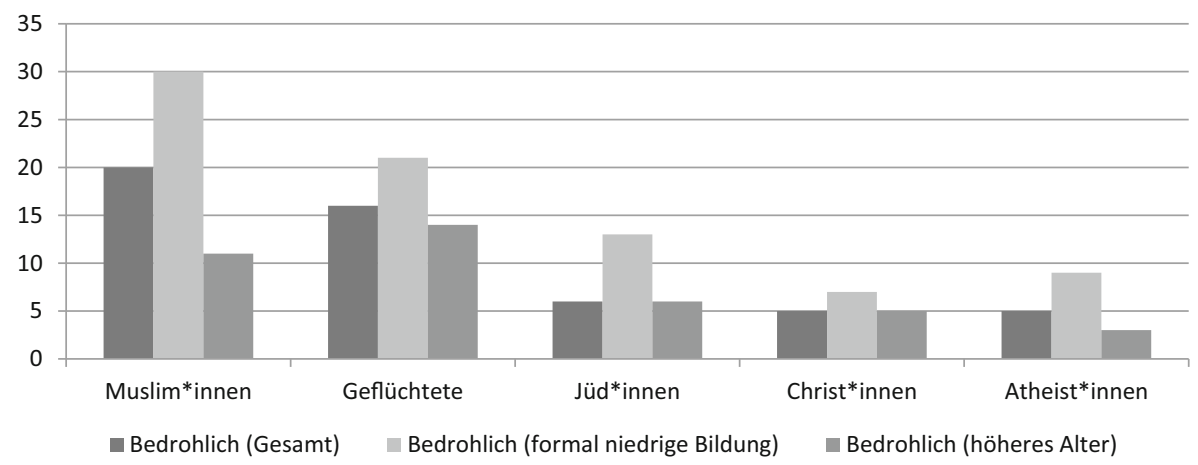

Abb. 2 Bedrohlichkeit von Religionen in Berlin. Frage: Wenn Sie an folgende Religionen und sozialen Gruppen denken: Als wie bedrohlich nehmen sie die folgenden sozialen Gruppen wahr? Anteil sehr bedrohlich/eher bedrohlich auf einer Antwortskala mit vier Antwortmöglichkeiten; Quelle: Berlin-Monitor $(N=2005)$

welche Gleichstellungsmaßnahmen für überzogen halten, scheint es sich eher um eine grundsätzliche als geschlechtsspezifische Haltung zu handeln. 
Tab. 2 Vernetzte Abwertung (Beziehungen zwischen verschiedenen Abwertungsformen)

\begin{tabular}{|c|c|c|c|c|c|c|c|c|c|c|c|}
\hline & $\mathrm{FF}$ & PAS & SAS & IAS & $\mathrm{AM}$ & FGf & $\mathrm{AZ}$ & DK & AG & Ras & Hom \\
\hline $\begin{array}{l}\text { Fremdenfeindlichke } \\
\text { (FF) }\end{array}$ & & - & - & - & - & - & - & - & - & - & - \\
\hline $\begin{array}{l}\text { Primärer } \\
\text { Antisemitismus } \\
\text { (PAS) }\end{array}$ & 0,37 & - & - & - & - & - & - & - & - & - & - \\
\hline $\begin{array}{l}\text { Sekundärer } \\
\text { Antisemitismus } \\
\text { (SAS) }\end{array}$ & 0,35 & 0,42 & - & - & - & - & - & - & - & - & - \\
\hline $\begin{array}{l}\text { Israelbezogener } \\
\text { Antisemitismus } \\
\text { (IAS) }\end{array}$ & 0,17 & 0,36 & 0,36 & - & - & - & - & - & - & - & - \\
\hline $\begin{array}{l}\text { Antimuslimische } \\
\text { Ressentiment } \\
\text { (AM) }\end{array}$ & 0,60 & 0,33 & 0,34 & 0,20 & - & - & - & - & - & - & - \\
\hline $\begin{array}{l}\text { Feindlichkeit } \\
\text { gegen } \\
\text { Geflüchtete } \\
\text { (FGf) }\end{array}$ & 0,65 & 0,28 & 0,35 & 0,19 & 0,58 & - & - & - & - & - & - \\
\hline $\begin{array}{l}\text { Antiziganismus } \\
\text { (AZ) }\end{array}$ & 0,47 & 0,23 & 0,30 & 0,22 & 0,45 & 0,46 & - & - & - & - & - \\
\hline $\begin{array}{l}\text { Soziale } \\
\text { Deklassierung } \\
\text { (DK) }\end{array}$ & 0,42 & 0,27 & 0,31 & 0,32 & 0,41 & 0,37 & 0,39 & - & - & - & - \\
\hline $\begin{array}{l}\text { Anti-Feminis- } \\
\text { mus (AF) }\end{array}$ & 0,34 & 0,23 & 0,25 & 0,21 & 0,31 & 0,33 & 0,28 & 0,33 & - & - & - \\
\hline $\begin{array}{l}\text { Klassischer } \\
\text { Rassismus } \\
\text { (Ras) }\end{array}$ & 0,31 & 0,28 & 0,29 & 0,17 & 0,29 & 0,29 & 0,24 & 0,27 & 0,31 & - & - \\
\hline $\begin{array}{l}\text { Homophobie } \\
\text { (Hom) }\end{array}$ & 0,20 & 0,20 & 0,20 & 0,18 & 0,26 & 0,19 & 0,10 & 0,09 & 0,29 & 0,26 & - \\
\hline $\begin{array}{l}\text { Transphobie } \\
\text { (TR) }\end{array}$ & 0,20 & 0,20 & 0,25 & 0,17 & 0,27 & 0,22 & 0,22 & 0,21 & 0,27 & 0,15 & 0,38 \\
\hline
\end{tabular}

Quelle: Eigene Darstellung auf Grundlage der Ergebnisse der Berlin-Monitor Umfrage; $N=2005$ Berliner*innen; Pearsons Produkt-Moment Korrelationen, $\mathrm{r}<0,05$

n. s. kein signifikanter Zusammenhang

knüpft als andere. Bereits angesprochen wurde die enge Verzahnung zwischen Haltungen gegenüber Muslim*innen und Geflüchteten. Diese Korrelation ragt aus den auffindbaren Beziehungsmustern heraus (Tab. 2). Beide Vorurteile überschneiden sich in einer Dimensionsanalyse, wie in den Korrelationsmustern mit der sozialen Abwertung von Sinte*zze und Rom*nja. ${ }^{21}$ Zudem bindet sich die Abwertung von Langzeitarbeitslosen statistisch an diese Aussagen an. Dieses Set an Vorurteilen kennzeichnet Muslim*innen als Fremdgruppen mit einer spezifischen Einordnung als kulturell fremd, oder normativ stark abweichend aus Sicht der Urteilenden. Soziale Deklassierung koppelt sich mit kulturellen Abwertungsmustern. Daneben

\footnotetext{
21 Die durchgeführte Dimensionsanalyse beruht auf einer Hauptkomponentenanalyse mit schiefwinkliger Rotation unter Vorgabe dreier Dimensionen.
} 


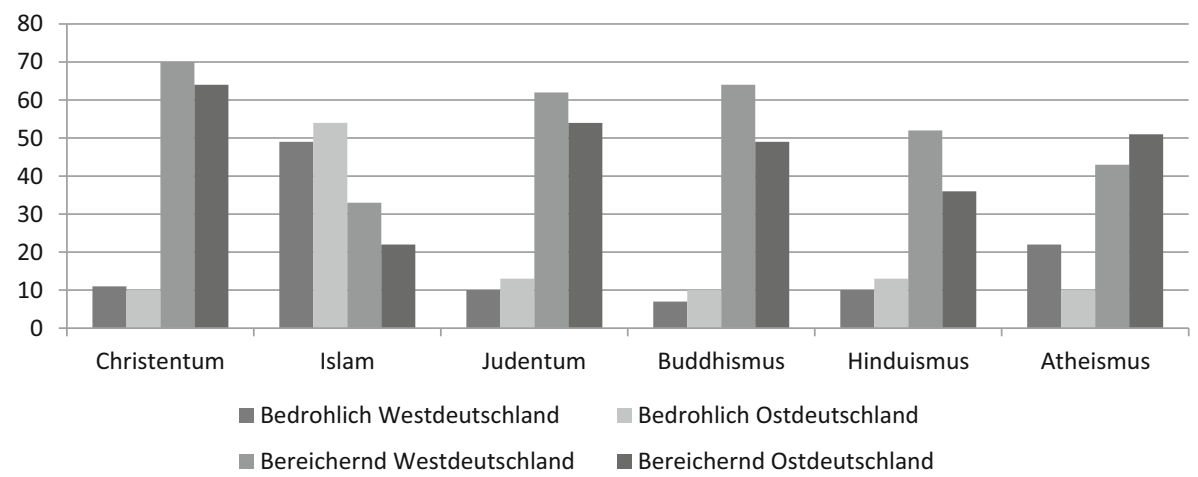

Abb. 3 Bedrohlichkeit und Bereicherung von Religionen in Deutschland. Frage: Wenn Sie an die Religionen denken, die es auf der Welt gibt: Als wie bedrohlich bzw. wie bereichernd nehmen sie die folgenden Religionen wahr? Anteil sehr bedrohlich/eher bedrohlich auf einer Antwortskala mit vier Antwortmöglichkeiten; Quelle: Religionsmonitor 2017; Basis: Bevölkerung Deutschland ( $N=1511)$; siehe Pickel 2019

korrelieren noch - wenig überraschend - die Aussagen zum Antisemitismus. Selbst wenn die Unterschiede zu den Korrelationen mit anderen Abwertungsitems relational sind, zeigen sie eine etwas stärkere Nähe in der Abwertung der beiden „Religionsgruppen“ auf: Manch eine Berliner*in, die antisemitische Ressentiments aufweist, besitzt auch Vorurteile gegenüber Muslim*innen. Interessant, spricht man heute von Fremdenfeindlichkeit, dann haben die Bürger*innen überwiegend Muslim*innen vor Augen.

Auch andere Abwertungen bündeln sich unterhalb einer gemeinsamen Menschenfeindlichkeit. Führt man die Einzelaussagen strukturierende Faktorenanalysen durch, dann findet sich eine Dreiteilung der Vorurteilsstrukturen. So lassen sich die Haltungen zu Geschlechtsidentitäten und Aspekte von Fremdenfeindlichkeit genauso voneinander unterscheiden, wie ein eigenständiges Syndrom des Antisemitismus zu identifizieren ist. ${ }^{22}$ Auf Geschlechtsidentitäten und sexuelle Orientierungen bezogene Aussagen liegen näher beieinander, als Haltungen gegenüber Langzeitarbeitslosen und Muslim*innen. Wenig überraschend wird Transsexualität bzw. Transgeschlechtlichkeit abgelehnt, wenn man Homo- und/oder Bisexualität als falsch empfindet. Die Vorurteile gegenüber Schwarzen Menschen, welche man als klassischen Rassismus bezeichnen kann (auch Diangelo 2018; Hasters 2019), machen wiederum eine eigenständige Dimension auf - und liegen etwas quer zu den anderen Formen der Ungleichwertigkeitsvorstellungen. Ebenfalls quer dazu ordnet sich statistisch die Haltung zu Geschlechtergerechtigkeit an. Sie steht mit allen Formen sozialer Abwertung in Verbindung, scheint aber eine Art generelle Skepsis gegen dieser ,modernistischen“ Maßnahme in Demokratien abzubilden. Insgesamt wird deutlich, dass unterhalb der Ebene einer von nicht wenigen Berliner*innen geteilten, in ihrer Ausrichtung breit gefächerten, gruppenbezogenen Menschenfeindlichkeit, bei einigen

22 Eine andere Interpretation der Aufteilung kann über die unterschiedliche Gruppenwahrnehmung nach Wärme oder Kompetenz geschehen (Fiske et al. 2002). Hier danken wir der Gutachter*in für ihren Hinweis. 
Berliner*innen eine Differenzierung der Vorurteile existiert. Anders gesagt: Nicht jeder lehnt alle anderen sozialen Gruppen ab, aber viele zumindest eine und auch nicht wenige alle Anderen.

\section{Bedrohungsgefühle - und ihre Wirkung auf Vorurteile und Ressentiments?}

\subsection{Bedrohungsängste - Angst vor wem?}

Eine unserer zentralen Thesen war nun, dass eine gefühlte Bedrohung durch eine soziale Gruppe zu deren Ablehnung beiträgt. Anschließend an diese Annahme der Integrated Threat Theory wollen wir für die beiden in gruppenbezogenen Vorurteilen und Ressentiments immer wieder besonders herausgehobenen religiösen Gruppen, Jüd*innen und Muslim*innen, diese These - auch in Relation zu konkurrierenden Erklärungsmöglichkeiten - testen. Hierzu wurde im Berlin-Monitor die Wahrnehmung von Bedrohung durch verschiedene soziale Gruppen gemessen. ${ }^{23}$ Insgesamt weist nur etwas mehr als jede vierte Berliner*in solche Gefühle auf. Am stärksten noch werden Muslim*innen (von einem Fünftel der Berliner*innen) als bedrohlich angesehen, knapp darauf Geflüchtete. Beide Bedrohungswahrnehmungen sind wieder stark untereinander verbunden, wie eine Binnenkorrelation der Bedrohungsgefühle von $r=0,68$ eindrucksvoll belegt. Die Gleichsetzung von Geflüchteten mit Muslim*innen setzt sich auch in der gefühlten Bedrohungslage fort (auch Pickel und Pickel 2019, S. 295-299; Pollack et al. 2014). Andere Gruppen fallen deutlich hinter diese Einschätzung zurück. Immerhin sind es noch die Jüd*innen, welche knapp vor Christ*innen und Atheist*innen liegen. (Abb. 2).

Das Gefühl der Bedrohung ist unter den Berliner*innen sozialstrukturell unterschiedlich verteilt. Während jüngere Menschen in der Regel eine niedrigere Furcht vor anderen sozialen Gruppen äußern, steigt diese Angst mit höherem Alter und einer formal niedrigeren Bildung in beachtlichem Ausmaß an. Dieser Effekt gilt noch am geringsten für die Christ*innen, aber bei der Angst vor den Muslim*innen erreicht das Gefühl einer Bedrohung $30 \%$ und gegenüber jüdischen Bürger*innen mit formal niedriger Bildung mehr als den doppelten Wert zum Durchschnitt. Folgt man den internationalen Diskussionen hierzu wäre es durchaus angebracht von einer Islamophobie zu sprechen (Bleich 2011; Halliday 1999; Helbling 2012), beschreibt diese Zahl doch eine furchtbezogene Ablehnung und Abgrenzung - deren objektive Gefährdung kaum nachgehalten werden kann. Hierfür können unterschiedliche Gründe angeführt werden. Neben einer tendenziell stärkeren Wahrnehmung eines Konkurrenzdrucks, sind nationalistische Positionen in älteren Altersgruppe und unter Personen mit niedriger Bildung häufiger vertreten. Auch das Gefühl weniger als den gerechten Anteil am Lebensstandard zu erhalten erweist sich als, allerdings nur schwach, Bedrohungsängste steigernd. Bedrohungsgefühle haben entsprechend

\footnotetext{
${ }^{23}$ Leider liegt eine Messung der Bedrohlichkeit nicht für alle Gruppen vor. Gründe sind Platzgründe im Fragebogen und die Annahme, dass für viele von Vorurteilen belegten Gruppen das Bedrohungsszenario vermutlich nicht passt.
} 
durchaus mit sozialen Lagen zu tun, sie sind aber keineswegs ein direktes Abbildung des sozialen Umfeldes. Insgesamt fallen die Bedrohungsängste für Berlin sowieso eher niedrig aus, vergleicht man sie zum Bertelmann Religionsmonitor. 2013 wie 2017 wurde danach gefragt, ob man andere Religionen als eher bedrohlich oder bereichernd für die eigene Gesellschaft ansieht (Pickel 2019, S. 82-83; auch Pickel et al. 2016). Die zustimmenden Antworten zu einem Bedrohungsgefühl liegen in Berlin durchweg unter dem Bundesschnitt. Dies kann neben der unterschiedlichen Zielpopulation auch an der unterschiedlichen Fragestellung in beiden Studien liegen. So wie im Bertelsmann Religionsmonitor eine Kombination von bedrohlich und bereichernd abgefragt wurde, richtete sich dort die Einschätzung auch auf die Religionen an sich - und nicht exakt deren Mitglieder. Eine Ähnlichkeit ist allerdings zwischen beiden Umfragen erkennbar: Die Reihenfolge der zugewiesenen Bedrohlichkeit. Während der Buddhismus, der Hinduismus, und das Christentum in deutlicher Mehrheit als bereichernd eingeschätzt werden und nur von einem Zehntel der Befragten als bedrohlich, sieht dies beim Judentum und beim Islam anders aus. Immerhin die Hälfte der Bürger*innen in West- und Ostdeutschland empfinden den Islam in einer Globalbewertung als bedrohlich. ${ }^{24}$ (Abb. 3).

Interessant ist, dass die Werte zwischen 2013 und 2017 im Bertelsmann Religionsmonitor faktisch keine Veränderung erfahren haben. Es scheint sich bei den Einschätzungen, speziell des Judentums und des Islams, um tiefer liegende Ressentiments zu handeln - die der „Flüchtlingskrise“ nicht bedurften, außer um zum visuell sichtbaren öffentlichen Thema zu werden (Pickel 2019, S. 83).

\subsection{Bedrohungsängste - und ihre Bedeutung für Ressentiments?}

Die Annahme ist nun, dass diese Ängste ein zentraler Faktor für Ressentiments gegenüber Mitgliedern der beiden Religionsgemeinschaften nach sich ziehen. Bereits ein einfaches Korrelationsmodel zeigt die Beziehungen zwischen Bedrohungsgefühlen und Vorurteilen den entsprechenden Gruppen gegenüber (bei Muslim*innen $(p=+0,41)$, bei Jüd*innen $(r=+0,32$ bis $r=+0,16$ bei Israelbezogenem Antisemitismus.)). Nun sind neben Bedrohungsängsten natürlich auch andere Erklärungen für antimuslimische Ressentiments und Antisemitismus denkbar. Wie im theoretischen Teil des Beitrages bereits diskutiert, können Effekte der relativen Deprivation (also einer auf ökonomische Konkurrenz zielende Intergruppeneffekt), ein geringer Bildungsstand, autoritäre Einstellungen und Nationalismus Vorurteile bestärken und hervorbringen. Ebenfalls nicht grundsätzlich auszuschließen ist eine unterschiedliche reale Bedrohung durch die verschiedenen Gruppen (Koopmans 2015, 2020). Diese reale Bedrohungslage ist nur im vorliegenden Modell nicht zu modellieren. Insgesamt kann allein ein multivariates Modell Auskunft über die relative Relevanz. der Bedrohungsängste für die Vorurteile geben. Zu diesem Zweck haben wir OLS-

\footnotetext{
${ }^{24}$ Diese Haltung schließt an die aus den 1990er Jahren stammende Aussage, eines Kampfes der Kulturen an (Huntington 1996). Dort wird auf die steigende Gefährdung kultureller Auseinandersetzung in Zukunft - und dabei u. a. speziell auf die Auseinandersetzung zwischen einem globalen Islam und dem Christentum hingewiesen.
} 
Regressionsmodelle auf antimuslimische Ressentiments und die drei Dimensionen des Antisemitismus durchgeführt. ${ }^{25}$

Beginnen wir mit den antimuslimischen Vorurteilen (oder Ressentiments). ${ }^{26}$ Es findet sich in der Tat eine Vielzahl an Einflussfaktoren, welche mit antimuslimischen Vorurteilen in Beziehung steht. Neben Bildungseffekten befördert vor allem eine rechte Positionierung auf der politische Ideologie messenden Links-RechtsSkala antimuslimische Vorurteile. Das Gefühl fehlender Responsivität der Politiker*innen (External Efficacy) erweist sich als nicht bedeutsam, anders als das Gefühl keinen Einfluss auf politische Entscheidungen (Internal Efficacy) zu besitzen. Scheinbar ist es nicht eine Politikerverdrossenheit, die Vorurteile hervorbringt, sondern das Gefühl eines Kontrollverlustes in der eigenen Gemeinschaft, der sich aus der Einschätzung einer geringen oder des Verlustes der eigenen Wirksamkeit auf Politik ergibt. ${ }^{27}$ Für die Relevanz von Persönlichkeitseigenschaften und Erziehung sprechen die Effekte autoritärer Einstellungen. Speziell die Dimensionen der autoritären Aggression und des Konventionalismus steigern antimuslimische Vorurteile. Diesen Effekten entgegen wirken - die in Berlin aufgrund der hohen Multikulturalität besonders ausgeprägten - Kontakte sowie die Existenz von Sozialkapital in Form von Sozialvertrauen (Putnam 2000). Die Indikatoren für die beide zuletzt genannten Theoriestränge scheinen - allen ihnen gegenüber kritischen Einwürfen zum Trotz - bereits auf einfache Art Vorurteile und Ressentiments zu reduzieren. Beachtenswert ist der, (wenn auch schwache) Vorurteile hemmende Einfluss von persönlicher Religiosität und einer guten wirtschaftlichen Situation (nach eigener Beurteilung). Die relative Deprivation besitzt keinen Einfluss. Sollte es keine Besonderheit von Berlin sein, sind soziale Ungleichheiten und ihre Wahrnehmung keine Erklärung für antimuslimische Vorurteile, was z.B. gegen den Ansatz der ökonomischen Gruppenbedrohung spricht. Für seine Relevanz spricht der Effekt der Einschätzung der eigenen Wirtschaftslage, während das Haushaltseinkommen ohne Effekt bleibt.

Integriert man Bedrohungsängste in das Erklärungsmodell (siehe Tab. 3), so weisen diese in Berlin auf Anhieb den stärksten Einfluss auf antimuslimische Ressentiments auf. Sieht man Muslim*innen und den Islam als bedrohlich oder gefährlich an, dann steigert sich Ressentiments gegenüber Muslim*innen in hohem Maße. Vergleicht man Modelle mit und ohne Einbezug der Bedrohungsangst (hier nicht gesondert ausgewiesen), steigert sich die Gesamterklärungskraft des Modelles bei Einbezug der Bedrohungsgefühle erheblich. Bedrohungsgefühle besitzen somit eine eigene - über eine Mediatorfunktion hinausgehende - Wirkungsmacht. Auch etwas anderes wird sichtbar: Im Vergleich reduzieren sich mit Einbezug des Bedrohungsgefühls zwar die Wirkungen der weiteren eingesetzten Erklärungsfaktoren, sie bleiben aber neben dem, nun dominanten Element der Bedrohungsangst, eigenständig

\footnotetext{
25 In den linearen Regressionen wurden zum Zweck der Vergleichbarkeit innerhalb des Datensatzes standardisierte Beta-Werte der Regression ausgewiesen.

${ }^{26}$ Für die Kausalanalyse antimuslimischer Vorurteile wurde aus beiden Items eine Likert-Skala zusammengesetzt.

27 Etwas überraschend kann speziell die häufig als Prädiktor genannte relative Deprivation im multivariaten Modell keinen Einfluss erzielen. Möglicherweise liegen die Ressentiments doch tiefer, als Eigeneinstufungen in der Gesellschaft.
} 
Tab. 3 Regression auf antimuslimische Ressentiments

\begin{tabular}{|c|c|c|}
\hline Regressionen Muslimfeindlichkeit & Modell $1^{\mathrm{a}}$ & Modell $2^{b}$ \\
\hline Wirtschaftliche Lage (besser) & $-0,08 * * *$ & $-0,08 * * *$ \\
\hline Abitur oder höher & $-0,06 * *$ & 0,02 \\
\hline Migrationshintergrund & $-0,05 *$ & $-0,07 * *$ \\
\hline Alltagskontakte: Viele Menschen anderer Herkunft & $-0,13 * * *$ & $-\mathbf{0 , 1 3} * * *$ \\
\hline Relative Deprivation & 0,02 & $-0,07 * *$ \\
\hline Alles in allem gesehen, kann man den meisten Menschen vertrauen & $-0,08 * * *$ & $-0,07 * *$ \\
\hline Alles in allem, kann man Politikern doch vertrauen & $-0,06 * *$ & $-0,08 * *$ \\
\hline External Efficacy & 0,00 & $\mathbf{0 , 0 6 *}$ \\
\hline Internal Efficacy & $\mathbf{0 , 1 0} * * *$ & 0,03 \\
\hline Links-Rechts-Selbsteinstufung & $\mathbf{0 , 1 8 * * *}$ & $0,16 * * *$ \\
\hline Politiker sind nur Marionetten (Verschwörungsmentalität) & $-0,01$ & 0,04 \\
\hline \multicolumn{3}{|l|}{ Autoritarismus } \\
\hline Unruhestifter sollen zu spüren bekommen, dass sie unerwünscht sind & $\mathbf{0 , 0 8} * * *$ & $\mathbf{0 , 0 7 * *}$ \\
\hline $\begin{array}{l}\text { Menschen sollten wichtige Entscheidungen den Führungspersonen } \\
\text { überlassen }\end{array}$ & 0,02 & 0,02 \\
\hline Bewährte Verhaltensweisen sollten nicht in Frage gestellt werden & $\mathbf{0 , 0 6 * *}$ & $\mathbf{0 , 0 6 * *}$ \\
\hline Religiosität-Skala & $-0,06 * *$ & 0,02 \\
\hline Religiöser Dogmatismus & - & - \\
\hline Katholik*Innen & $-0,03$ & 0,03 \\
\hline Protestant $*$ Innen & $-0,01$ & $0,09 * *$ \\
\hline Atheist*Innen & $-0,06$ & $0,09 * *$ \\
\hline Einschätzung der Bedrohlichkeit: Muslime & $\mathbf{0 , 3 2} * * *$ & - \\
\hline Konstante & $1,57 * * *$ & $1,94 * * *$ \\
\hline$R^{2}$ & 0,40 & 0,21 \\
\hline Korrigiertes $R^{2}$ & 0,39 & 0,21 \\
\hline Modellsignifikanz. & 0,00 & 0,00 \\
\hline
\end{tabular}

Anmerkungen: Eigene Darstellung auf Grundlage zweier OLS-Regressionsmodelle; Ausgewiesen sind die Beta-Werte, mit Ausnahme der Konstante; Datengrundlage: 2005 Befragte aus Berlin; Daten gewichtet. Paarweiser Fallausschluss

Abhängige Variablen:

${ }^{a}$ Muslimfeindlichkeit, zusammengesetzt aus den Items „Die Anzahl der Muslime in Deutschland ist zu hoch“ und „Muslimen sollte die Zuwanderung nach Deutschland untersagt werden“;

${ }^{\mathrm{b}}$ Einschätzung der Bedrohlichkeit: Muslime

Signifikanzniveaus: signifikante Werte schwarz markiert; $*=p<0,05 ; * *=p<0,01 ; * * *=p<0,001$

als signifikante Einflussfaktoren bestehen: Antimuslimische Ressentiments sind ein vielfältig beeinflusstes Phänomen, in dem die Angst vor der Referenzgruppe der Muslim*innen eine dominante - unabhängige - Bedeutung besitzt. Dieser Befund steht etwas im Gegensatz zu der Annahme, dass Bedrohungsgefühle in entsprechenden Modellen eine reine Mediatorfunktion besitzen. Wäre dies der Fall, hätten nach Einführung der Bedrohungsgefühle die alternativen Effekte weitgehend verschwinden müssen und der Gesamtkoeffizient wäre stabil geblieben. Dies ist aber, wie gerade gesagt, nicht der Fall. Gleichwohl ist diese Annahme einer Mediatorfunktion nicht vollständig falsch. Eine parallele Regressionsanalyse auf Bedrohungsgefühle durch Muslim*innen zeigt ein ähnliches, wenn auch nicht deckungsgleiches, Erklärungs- 
Tab. 4 Regression auf antisemitische Ressentiments

\begin{tabular}{|c|c|c|c|}
\hline Regressionen Antisemitismus & Modell $1^{\mathrm{a}}$ & Modell $2^{b}$ & Modell $3^{c}$ \\
\hline Wirtschaftliche Lage & $-0,02$ & $-0,04$ & $-0,06 * *$ \\
\hline Abitur oder höher & $-0,01$ & $-0,04$ & $-0,12 * * *$ \\
\hline Migrationshintergrund & $0,13 * * *$ & $-0,06 *$ & $0,06 *$ \\
\hline Alltagskontakte: Viele Menschen anderer Herkunft & $-0,03$ & $-0,04$ & $\mathbf{0 , 0 8} * *$ \\
\hline Relative Deprivation & 0,01 & $-0,06 *$ & $-0,03$ \\
\hline $\begin{array}{l}\text { Alles in allem gesehen, kann man den meisten Menschen } \\
\text { vertrauen }\end{array}$ & $-0,05 *$ & 0,00 & $-0,01$ \\
\hline Alles in allem, kann man Politikern doch vertrauen & 0,04 & 0,03 & $-0,04$ \\
\hline External Efficacy & 0,00 & $\mathbf{0 , 0 6 *}$ & $\mathbf{0 , 0 6 *}$ \\
\hline Internal Efficacy & $\mathbf{0 , 0 8} * *$ & $0,06^{*}$ & 0,02 \\
\hline Links-Rechts-Selbsteinstufung & $0,11 * * *$ & $\mathbf{0 , 1 5 * * *}$ & $-0,02$ \\
\hline $\begin{array}{l}\text { Politiker sind nur Marionetten (Verschwörungsmentali- } \\
\text { tät) }\end{array}$ & $0,18 * * *$ & $0,18 * * *$ & $0,20 * * *$ \\
\hline \multicolumn{4}{|l|}{ Autoritarismus } \\
\hline $\begin{array}{l}\text { Unruhestifter sollen zu spüren bekommen, dass sie uner- } \\
\text { wünscht sind }\end{array}$ & $\mathbf{0 , 0 5 *}$ & $0,09 * * *$ & 0,02 \\
\hline $\begin{array}{l}\text { Menschen sollten wichtige Entscheidungen den Füh- } \\
\text { rungspersonen überlassen }\end{array}$ & 0,00 & 0,00 & $\mathbf{0 , 0 7 * *}$ \\
\hline $\begin{array}{l}\text { Bewährte Verhaltensweisen sollten nicht in Frage gestellt } \\
\text { werden }\end{array}$ & $0,09 * * *$ & $\mathbf{0 , 0 8} * *$ & $0,06 * *$ \\
\hline Religiosität-Skala & $-0,06^{*}$ & $-0,02$ & 0,01 \\
\hline Religiöser Dogmatismus & - & - & - \\
\hline Katholik*Innen & 0,00 & $-0,04$ & $-0,14 * * *$ \\
\hline Protestant $*$ Innen & 0,04 & $-0,02$ & $-0,09 *$ \\
\hline Atheist*Innen & 0,00 & 0,00 & $-0,09 *$ \\
\hline Muslim*Innen & $0,14 * * *$ & 0,01 & $0,10 * *$ \\
\hline Einschätzung der Bedrohlichkeit: Juden & $0,15 * * *$ & $0,09 * * *$ & $0,11 * * *$ \\
\hline Konstante & 0,03 & 0,42 & $\mathbf{0 , 9 4} * *$ \\
\hline$R^{2}$ & 0,23 & 0,23 & 0,27 \\
\hline Korrigiertes $R^{2}$ & 0,22 & 0,22 & 0,26 \\
\hline Modellsignifikanz & 0,00 & 0,00 & 0,00 \\
\hline
\end{tabular}

Anmerkungen: Eigene Darstellung auf Grundlage dreier OLS-Regressionsmodelle; Ausgewiesen sind die Beta-Werte, mit Ausnahme der Konstante; Datengrundlage: 2005 Befragte aus Berlin; Daten gewichtet. Paarweiser Fallausschluss

Abhängige Variablen:

${ }^{a}$ Auch heute noch ist der Einfluss der Juden zu groß

${ }^{b}$ Reparationsforderungen an Deutschland nutzen oft gar nicht den Opfern, sondern einer Holocaust-Industrie von findigen Anwälten;

${ }^{c}$ Israels Politik in Palästina ist genauso schlimm wie die Politik der Nazis im zweiten Weltkrieg Signifikanzniveaus: signifikante Werte schwarz markiert; $*=p<0,05 ; * *=p<0,01 ; * * *=p<0,001$

muster. Überlappungen in den Prädiktoren sind also gegeben, allerdings verbleiben vielfältige eigenständige Effekte. (Tab. 4).

Somit besitzen Bedrohungsgefühle auf der einen Seite eine Mediatorfunktion, allerdings entfalten sie für antimuslimische Haltungen einen eigenständigen (emotionalen) Effekt, der neben grundsätzlich vorhandene Ressentiments gegenüber Mus- 
lim*innen tritt. Denn diese existiere auch, wie die noch beachtliche fehlende Erklärungsvarianz für die gemessenen Vorurteile belegt. So beruhen Vorurteile gegenüber Muslim*innen weder allein auf Ängsten, noch kann dies als Entschuldigung für Vorurteile und gar ablehnende Verhaltensweisen genutzt werden. Gleichzeitig weisen aber nicht nur Rassist*innen Vorurteile gegenüber Muslim*innen auf, was für die Prävention gegenüber einer Ausbreitung antimuslimischer Ressentiments durchaus Wege aufzeigt. Speziell, wenn man die Bedrohungsgefühle als weitgehend symbolische Bedrohungen und nicht als realistische Bedrohungen versteht. Und so widerspricht die Betrachtung der Effekte der Regression (zumindest in weiten Teilen) Annahmen, dass sich antimuslimische Vorurteile aus realen Bedrohungen speisen (Koopmans 2020). Wäre dies der Fall, dürften nicht Einstellungen wie Autoritarismus, rechte Ideologie oder Kontakthäufigkeit die stärksten Faktoren in der Analyse sein.

Das Ergebnis für antisemitische Einstellungen entspricht an vielen Stellen dem Ergebnis für die antimuslimischen Ressentiments. ${ }^{28}$ Auch dort befördern eine rechte ideologische Positionierung und eine fehlende Selbstwirksamkeit auf der politischen Ebene antisemitische Einstellungen. Hinzu treten nun aber der Einfluss einer Verschwörungsmentalität, sowie ein Effekt aufgrund eines Migrationshintergrundes (Rees und Lamberty 2019, S. 204, 209-211). Da gleichzeitig eine größere Kontaktbreite der Personen kaum mehr etwas gegen Antisemitismus bewirkt, haben wir es im Kern mit einem doch anderem Erklärungsmodell als bei antimuslimischen Ressentiments zu tun. Aufgrund der geringen Zahl an Jüd*innen in Deutschland ist der direkte Kontakt mit Jüd*innen im Lebensalltag generell sehr gering. Dazu trägt bei, dass Jüd*innen auf der Straße zusätzlich selten als solche identifiziert werden. Im Kontext der fehlenden Kontakte bilden sich Bürger*innen auf anderem Wege eine Meinung über und Haltung zu Jüd*innen. Da zudem die Zahl der parasozialen Kontakte (über mediale Berichterstattung) begrenzt ist, speisen sich Vorurteile gegenüber Jüd*innen in wesentlichem Maß aus Hörensagen sowie Verschwörungstheorien. Letztere drücken sich in den Bedrohungsängsten gegenüber Jüd*innen aus. Diese besitzen, wie bereits die Ängste vor Muslim*innen bei den antimuslimischen Ressentiments, einen signifikanten Einfluss auf antisemitische Ressentiments aller Richtungen. Dies gilt für primären, sekundären wie israelbezogenen Antisemitismus. Allerdings fällt der Einfluss der Bedrohungsängste im Vergleich zum Einfluss der Ängste vor Muslim*innen deutlich schwächer aus. Ob in Umwegkommunikation oder direkt, eine verschwörungstheoretisch untermauerte Furcht vor Jüd*innen als Drahtzieher einer Weltverschwörung ist in Deutschland und in Berlin noch immer vorhanden (vgl. Decker et al. 2018, S. 190-199; Zick et al. 2019, S. 102-108). Dies wird auch daran deutlich, dass die Verschwörungsmentalität für antimuslimische Ressentiments ohne jeglichen signifikanten statistischen Einfluss ist. Während bei Muslim*innen teilweise reale Ängste und eine wahrgenommene realistische Bedro-

\footnotetext{
${ }^{28}$ Für die Erklärung der antisemitischen Einstellungen wurde auf eine Bündelung der Indikatoren zu einer Dimension antisemitische Einstellungen genauso verzichtet, wie auf die für antimuslimische Ressentiments dargestellte Parallelbetrachtung der Bedrohungsgefühle. Zum einen halten wir die Differenzierung der Ergebnisse nach Dimensionen antisemitischer Einstellungen für wichtiger, zum anderen ist auch der Bedrohungseffekt bei antisemitischen Einstellungen deutlich geringer, so dass eine Differenzierung in der Darstellung nur unübersichtlich geworden wäre.
} 
hung Einfluss auf die Haltungen besitzen, reflektiert eine geäußerte Bedrohung durch Jüd*innen eher eine Übertragung eines bestehenden Stereotyps und Ressentiments. Anders gesagt könnte es sein, dass antimuslimische Vorurteile oft eine Folge von Ängsten ihnen gegenüber ist, während die Äußerung einer gefühlten Bedrohung durch Jüd*innen die Folge grundlegender antisemitischer Ressentiments ist.

Wir finden noch einen weiteren Effekt. So erweist sich ein Migrationshintergrund und die Zugehörigkeit zur Glaubensgruppe der Muslim*innen, oder besser zu Migrant*innen mit muslimischen Hintergrund, als Verstärker für antisemitische Einstellungen. Speziell beim israelbezogenen Antisemitismus kommt es zu merklichen Unterschieden zwischen den verschiedenen Religionszugehörigkeiten. Dieses Phänomen des muslimischen Antisemitismus hat in jüngerer Zeit beachtliche öffentliche Aufmerksamkeit erfahren und wird seitens rechtspopulistischer Parteien und Politiker*innen gerne in antimuslimischen Kampagnen instrumentalisiert. Dabei wird eine der islamischen Religion innewohnende kulturelle antisemitische Prägung propagiert, die durch Umwelterfahrungen nicht veränderbar sei. Diese Einschätzung ist ethnisierend, blendet sie doch Anpassungseffekte an eine neue Umwelt vollständig aus (siehe Cakir 2014; Shooman 2014). ${ }^{29}$ Auch ein anderer Grund revidiert einen „,religiösen Effekt““. So können kulturelle Prägungen aus den Herkunftsgebieten bei Muslim*innen mit Migrationshintergrund, Grund für die ablehnende Haltung gegenüber Jüd*innen sein. Solche Einstellungen sind sozial gebunden, also vor anderen Erfahrungshorizonten veränderbar. Zudem gilt es zu berücksichtigen, dass es sich um eine deutliche Minderheit handelt, die eine religiöse Ablehnung von Jüd*innen aufgrund der Religion äußert (Tab. 5), sich bedroht fühlt (30\%) oder antisemitische Einstellungen aufweist. Die Mehrheit der Muslim*innen in Berlin steht Jüd*innen weitgehend neutral bis wohlwollend gegenüber.

Trotzdem kann die eigene Religiosität ein Grund für die Einstellungen sein, z. B., wenn es sich um eine dogmatischere Religiosität mit antijüdischer Ausrichtung handelt. So geben immerhin 15\% der im Berlin-Monitor befragten Muslim*innen an,

Tab. 5 Dogmatismus in den Religionen

\begin{tabular}{lll}
\hline & Christ*innen & Muslim*innen \\
\hline Es gibt nur eine Auslegung der Bibel/des Koran und alle Christen/ & 7 & 44 \\
Muslime müssen sich daran halten & 17 & 56 \\
Die Regeln der Bibel/des Korans sind mir wichtiger als die deutschen & 5 & 34 \\
Gesetze & 17 & 52 \\
Meine Religion gibt mir vor Juden zu misstrauen und sie abzulehnen & 1 & 13 \\
& 1 & 15 \\
Ich wäre bereit meine Überzeugungen auch mit Gewalt durchzusetzen & 1 & 0 \\
& 2 & 5 \\
\hline
\end{tabular}

Quelle: Berlin-Monitor 2019; Basis: Berlin $(N=2005)$; Werte in Prozent; erster Wert stimme voll und ganz zu, zweiter Wert stimme voll und ganz zu + stimme eher zu auf einer Skala mit vier Antwortmöglichkeiten

29 Und dies nicht nur, weil das besonders auffällige Zusammenkommen von antisemitischen und antimuslimischen Vorurteilen bei Anhänger*innen von rechtspopulistischen und rechtsradikalen Parteien deutlich im Widerspruch zu einer angeblichen Sorge um Jüd*innen in Deutschland steht (siehe hierzu Decker et al. 2018, S. 191). Vergleichsuntersuchungen der muslimischen Bevölkerung in Deutschland und anderen europäischen Staaten gegenüber der Türkei zeigen massive Unterschiede (Pickel 2019, S. 61). 
dass ihre Religion ihnen vorgibt, Jüd*innen zu misstrauen und sie abzulehnen. Dieser „Antijudaismus“ kommt in Berlin nur bei einem Prozent der Christ*innen zum Tragen. Überhaupt steht unter den Muslim*innen ihre Religion höher im Kurs und dient häufiger als bei den Christ*innen als Richtlinie für den Lebensalltag. Mehr als die Hälfte unter ihnen hält die Regeln des Koran für wichtiger als die deutschen Gesetze und sieht eine stark auf die Ursprungsschrift begrenzte Deutung des Korans. ${ }^{30}$ Diese dogmatische Position teilt nicht einmal jede vierte Christ*in. Die beachtliche Zahl von Berliner Muslim*innen, die diesen Aussagen voll und ganz zustimmen, verweisen auf eine tragende Rolle der Religion für ihr Leben. Dies unterscheidet viele Muslim*innen in Berlin von ihren christlichen und konfessionslosen Nachbar*innen. Allerdings muss man die Relationen im Blick behalten: Sowie die Zahl derjenigen, welche zustimmt, gerade einmal ein Achtel der in Berlin lebenden Muslim*innen ausmacht, so zeigt sich bei einer weiteren Frage eine deutliche Distanz auf, Gewalt zur Durchsetzung ihrer religiösen Vorstellungen einsetzen zu wollen. Gleichwohl ist die höhere Zahl dogmatisch denkender Muslim*innen für antisemitische Einstellungen von Relevanz - besteht doch ein empirischer Wirkungszusammenhang. Diesen strukturellen Zusammenhang finden wir auch bei dogmatischen Christ*innen, nur ist eben der prozentuale Anteil der dogmatischen Christ*innen unter allen Christ*innen in Berlin niedriger (Tab. 6; auch Pickel et al. 2020). ${ }^{31}$

Tab. 6 Dogmatismus in den Religionen und antisemitische Einstellungen

\begin{tabular}{llll}
\hline & $\begin{array}{l}\text { Primärer } \\
\text { Antisemitismus }\end{array}$ & $\begin{array}{l}\text { Sekundärer } \\
\text { Antisemitismus }\end{array}$ & $\begin{array}{l}\text { Israelbezogener } \\
\text { Antisemitismus }\end{array}$ \\
\hline $\begin{array}{l}\text { Es gibt nur eine Auslegung der Bibel und } \\
\text { alle Christen müssen sich daran halten }\end{array}$ & $+0,26$ & $+0,22$ & $+0,14$ \\
$\begin{array}{l}\text { Es gibt nur eine Auslegung des Koran und } \\
\text { alle Muslime müssen sich daran halten }\end{array}$ & $+0,12$ & $+0,18$ & $+0,29$ \\
$\begin{array}{l}\text { Die Regeln der Bibel sind mir wichtiger als } \\
\text { die deutschen Gesetze. (Christ*innen) }\end{array}$ & $+0,10$ & $\mathrm{n} . \mathrm{s}$. & $+0,08$ \\
$\begin{array}{l}\text { Die Regeln des Korans sind mir wichtiger } \\
\text { als die deutschen Gesetze. (Muslim*innen) }\end{array}$ & $+0,33$ & $+0,26$ & $+0,25$ \\
$\begin{array}{l}\text { Meine Religion gibt mir vor Juden zu miss- } \\
\text { trauen und sie abzulehnen. (Christ*innen) }\end{array}$ & $+0,13$ & $+0,08$ & $+0,11$ \\
$\begin{array}{l}\text { Meine Religion gibt mir vor Juden zu miss- } \\
\text { trauen und sie abzulehnen. (Muslim*innen) }\end{array}$ & $+0,21$ & $+0,36$ & $+0,30$ \\
$\begin{array}{l}\text { Ich wäre bereit meine Überzeugungen auch } \\
\text { mit Gewalt durchzusetzen. (Christ*innen) }\end{array}$ & $+0,08$ & $+0,17$ & $+0,19$ \\
$\begin{array}{l}\text { Ich wäre bereit meine Überzeugungen auch } \\
\text { mit Gewalt durchzusetzen. (Muslim*innen) }\end{array}$ & $\mathrm{n} . \mathrm{s}$. & $\mathrm{n} . \mathrm{s}$. & $\mathrm{n} . \mathrm{s}$.
\end{tabular}

Quelle: Berlin-Monitor 2019; Basis: Berlin $(N=2005)$; Pearsons Produkt-Moment Korrelationen; Muslim*innen $(n=172)$; Christ*innen $(n=683)$; alle Zusammenhänge signifikant bei $p<0,05$

\footnotetext{
30 Diese Messung ist eine Variation einer als fundamentalistisch eingestuften Antwortvorgabe bei Koopmans (2017, S. 168). Die Werte in Berlin bleiben unter den bundesweit ermittelten Ergebnissen von Koopmans (2017, S. 174).

31 Hier muss man beachten, dass aufgrund der unterschiedlichen Gruppengrößen in absoluten Zahlen die Zahl der dogmatischen oder fundamentalistischen Christ*innen nicht mehr unter den Muslim*innen liegt, speziell im Bundesgebiet.
} 
$\mathrm{Zu}$ diesem Niveauunterschied mit seinen Auswirkungen treten zwei interessante Effekte: Zum einen fallen die Zusammenhänge zwischen antisemitischen Einstellungen und einer religiösen Begründung unter Muslim*innen wesentlich höher aus als unter den Christ*innen. Bei aller sozialer Relativierung wirkt sich eine dogmatische Religionszugehörigkeit ungünstig auf die Haltung zu Jüd*innen aus. Entsprechend früher Überlegungen in der Theorie des Autoritarismus (Adorno 1973) wirkt hier eine tiefer gehende (dogmatische verstandene) Religiosität förderlich für Vorurteile. Mindestens ebenso bemerkenswert ist aber ein zweites Ergebnis. So besteht zwischen den antisemitischen Einstellungen bei Muslim*innen und der Bereitschaft seine (teils religiösen) Interessen mit Gewalt durchzusetzen kein signifikanter Zusammenhang. Einfach gesagt: Antisemitische Einstellungen sind unter Muslim*innen weiter verbreitet und werden auch religiös begründet, sie münden aber (wenn man diese Selbstangabe ernst nimmt) nicht zwingend in antisemitische Handlungen. Dies sieht bei den wenigen Christ*innen mit gleicher Einstellung anders aus, sind diese eindeutig häufiger bereit Gewalt zur Durchsetzung ihrer Haltungen einzusetzen.

Fassen wir zusammen: Antisemitische Einstellungen speisen sich in Berlin aus unterschiedlichen Quellen. Dabei sind die Begründungen für antisemitische Einstellungen sichtbar unterschiedlich zu antimuslimischen Vorurteilen. Resultieren sie auf der einen Seite aus Herkunft und möglicherweise religiöser Ablehnung, ist auf der anderen Seite bei anderen Personen eine rechte Ideologie und eine tief verankerte Verschwörungsmentalität die Triebkraft für Einstellungsantisemitismus. Letztere Aspekte sind zusammen mit autoritären Einstellungen ein deutlich stärkerer Prädiktor von antisemitischen Einstellungen als die Zugehörigkeit zu einer muslimischen Glaubensgemeinschaft. Dort ist es nur die Minderheit der der Dogmatiker und Fundamentalisten (und nicht einmal alle von diesen), welche entsprechende Vorurteile aufweisen. Selbst diese sind allen Ergebnissen zufolge nur in sehr begrenzter Form gewaltbereit. Gleichwohl ist somit unter den religiöseren und öfter dogmatisch religiösen Muslim*innen ein Potential für Antisemitismus. Wenn, dann begründen sie diese Vorurteile häufiger religiös, während Nichtmuslim*innen eher eine völkische Ideologie zum Ausdruck bringen. Es ist anzunehmen, dass diese Ergebnisse im Bundesgebiet nicht grundsätzlich anders sind.

\section{Bedrohungswahrnehmungen, Ressentiments und Wahl von Rechtspopulisten}

Nun ist das Zustandekommen von gruppenbezogenen Vorurteilen und Ressentiments die eine Sache, eine andere ist, welche politische Relevanz Vorurteile entfalten. Zum einen stellt sich die Frage nach den Auswirkungen von Vorurteilen auf die Demokratie. Ein einfacher Blick auf eine Korrelationsmatrix zeigt Zusammenhänge zu antidemokratischen Einstellungsmustern und zur Demokratiezufriedenheit, die sich teilweise unterscheiden. Die Tabelle zeigt aber auch: Vorurteile sind eher unproduktiv für eine liberale, pluralistisch ausgerichtete Demokratie. Beginnen wir auf der Ebene von Demokratiebeurteilungen, wo sich Legitimität und Effektivitätsbeurteilung treffen - der Zufriedenheit mit der aktuellen Demokratie (Pickel und Pickel 2020). Eine Unzufriedenheit mit der aktuellen Demokratie wird zentral von der 
Ablehnung von Geflüchteten, Muslim*innen und antisemitischen Einstellungen getragen. Auch soziale Deklassierung, Antiziganismus und der klassische Rassismus sind mit einer antidemokratischen Grundhaltung verzahnt. Andere gruppenbezogene Vorurteile stehen hinter diesen Zusammenhängen zurück. Wenn sie mit der gegenwärtigen Demokratie nicht zufrieden sind, dann liegt dies bei den meisten Berliner*innen an Ängsten, die stark mit Geflüchteten und dem Umgang mit den Fluchtbewegungen sowie diesbezüglichen spezifischen wie unspezifischen Ängsten in Beziehung stehen. Auch tiefer liegende Vorurteile befördern allerdings eine Distanzhaltung zur aktuellen Demokratie. (Tab. 7).

Demokratietheoretisch bedeutsamer sind die Beziehungen zu einer klar antidemokratischen Haltung, wie sie die Befürwortung eines starken Führers operationalisiert. Das Ergebnis fällt kaum anders aus als bei der Demokratiezufriedenheit, und ist sogar etwas deutlicher in seinen Konturen: Vorurteile, autoritäre Einstellungen sowie der Wunsch nach autoritärer Herrschaft scheinen Hand in Hand zu gehen. Allein wieder die Haltungen zu Gleichstellung, Homophobie und Transphobie fallen in der Stärke der Beziehungen ein wenig hinter die anderen Vorurteile zurück. Doch in Relation zu einem ,starken Führer“ weisen sie immer noch signifikante

Tab. 7 Korrelationen zwischen Vorurteilen und (anti-)demokratischen Einstellungen

\begin{tabular}{lll}
\hline & $\begin{array}{l}\text { Wir sollten einen Führer haben, der Deutsch- } \\
\text { land mit starker Hand zum Wohle aller regiert }\end{array}$ & $\begin{array}{l}\text { Zufriedenheit mit der } \\
\text { Demokratie in } \\
\text { Deutschland }\end{array}$ \\
\hline $\begin{array}{l}\text { Primärer Antisemitis- } \\
\text { mus }\end{array}$ & $\mathbf{+ 0 , 3 4}$ & $-0,16$ \\
$\begin{array}{l}\text { Sekundärer Antisemi- } \\
\text { tismus }\end{array}$ & $+0,23$ & $-0,20$ \\
$\begin{array}{l}\text { Israelbezogener Anti- } \\
\text { semitismus }\end{array}$ & $+0,18$ & $-0,18$ \\
$\begin{array}{l}\text { Gefühl einer Be- } \\
\text { drohung durch }\end{array}$ & $\mathbf{+ 0 , 2 2}$ & $\mathbf{0 , 2 2}$ \\
Jüd*innen & & \\
Gefühl einer Bedro- & $\mathbf{+ 0 , 2 3}$ & \\
hung durch Mus- & & $\mathbf{- 0 , 2 7}$ \\
lim*innen & & \\
Antimuslimische Res- & $\mathbf{+ 0 , 3 3}$ & $\mathbf{0 , 2 5}$ \\
sentiments & & \\
Ablehnung von Ge- & $\mathbf{+ 0 , 2 8}$ & $\mathbf{- 0 , 2 7}$ \\
flüchteten & & \\
Antiziganismus & $\mathbf{+ 0 , 2 6}$ & $-0,14$ \\
$\begin{array}{l}\text { Transphobie } \\
\text { Homophobie }\end{array}$ & $\mathbf{+ 0 , 1 9}$ & n.s. \\
Soziale Deklassierung & $\mathbf{+ 0 , 1 5}$ & $-0,07$ \\
Klassischer Rassismus & $\mathbf{+ 0 , 2 6}$ & $-0,19$ \\
$\begin{array}{l}\text { Ablehnung von Gleich- } \\
\text { stellung }\end{array}$ & $\mathbf{+ 0 , 2 6}$ & $-0,10$ \\
\hline & $\mathbf{+ 0 , 1 1}$ & n.s. \\
\hline
\end{tabular}

Quelle: Eigene Berechnungen, Pearsons R Produkt-Moment-Korrelationen; ausgewiesen standardisierte Korrelationskoeffizienten n. s. nicht signifikanten Effekte bei $p>0,05$ 
Zusammenhänge auf. Fasst man diese Ergebnisse zusammen, so sind Vorurteile und Ressentiments ein Baustein antidemokratischer Einstellungen und Haltungen. Eine antipluralistische Haltung ist häufig auch eine antidemokratische Haltung. Damit wird dann die Ablehnung und Abwertung anderer Sozialgruppen zu einem demokratietheoretischen Problem, dass aus dem Spektrum reiner Unzufriedenheit mit der Tagespolitik heraustritt. Unter den abgelehnten Gruppen sind die religiös identifizierbaren diejenigen, welche am stärksten mit einer antidemokratischen Haltung in Bezug stehen. Die gefühlte Bedrohung durch Jüd*innen und Muslim*innen ist ein tragender Faktor für grundsätzliche Offenheit gegenüber Autokratie, wie auch einer konkreten Unzufriedenheit mit der gegenwärtigen Demokratie.

Diese Aktualität der gleichzeitigen Ablehnung von Muslim*innen und Geflüchteten gibt einen Hinweis auf deren Relevanz für die aktuellen rechtspopulistischen

Tab. 8 Regressionsergebnisse für gruppenbezogene Ressentiments nach Parteipräferenz

\begin{tabular}{|c|c|}
\hline Gruppenbezogene Ressentiments nach Parteipräferenz & $\begin{array}{l}\text { Option Voice } \\
\text { Wahl der AfD }\end{array}$ \\
\hline Primärer Antisemitismus & 0,10 \\
\hline Sekundärer Antisemitismus & 0,23 \\
\hline Israelbezogener Antisemitismus & $-0,32$ \\
\hline Gefühl einer Bedrohung durch Juden*Jüdinnen & $-0,51$ \\
\hline Gefühl einer Bedrohung durch Muslim*Innen & $0,71 *$ \\
\hline Antimuslimische Ressentiments & 0,35 \\
\hline Gefühl einer Bedrohung durch Geflüchtete & $0,67 *$ \\
\hline Ablehnung von Geflüchteten & 0,72 \\
\hline Antiziganismus & 0,26 \\
\hline Transphobie & $-0,05$ \\
\hline Homophobie & $-0,34$ \\
\hline Soziale Deklassierung & 0,12 \\
\hline Klassischer Rassismus & 0,05 \\
\hline Ablehnung von Gleichstellung & 0,04 \\
\hline Relative Deprivation & 0,00 \\
\hline Bildungsstand (formal niedrige Bildung) & $-0,21$ \\
\hline Einschätzung wirtschaftliche Lage (gut) & $-0,35$ \\
\hline Politische Ideologie (rechts) & $0,66^{* *}$ \\
\hline Verschwörungsmentalität & $\mathbf{0 , 5 7 *}$ \\
\hline Autoritarismus (Konventionalismus) & $0,46^{* *}$ \\
\hline Konstante & $-8,40 * * *$ \\
\hline Nagelkerke $R^{2}$ & 0,44 \\
\hline$N$ & 1244 \\
\hline Modellsignifikanz & $\mathbf{0 , 0 0}$ \\
\hline
\end{tabular}

Anmerkungen: Eigene Darstellung auf Grundlage zweier logistischer Regressionsmodelle; ausgewiesen ist jeweils der Regressionskoeffizient B; Datengrundlage: 2005 Befragte aus Berlin; Daten gewichtet. Paarweiser Fallausschluss

Abhängige Variablen: Parteipräferenz abgeleitet aus der Antwort auf die Frage „Unabhängig davon, ob Sie wahlberechtigt sind oder nicht: Wenn am nächsten Sonntag Bundestagswahl wäre, welche Partei würden Sie dann wählen?"

Signifikanzniveaus: signifikante Werte schwarz markiert; $*=p<0,05 ; * *=p<0,01 ; * * *=p<0,001$ 
Erfolge (Mudde und Kaltwasser 2017; Quendt 2019). Es stellt sich die Frage, ob auch im eher multikulturellen Berlin entsprechende Vorurteile das Wahlverhalten in Richtung rechtspopulistischer Parteien verschieben. Mehrere Befunde in der Wahlforschung (Hambauer und May 2018; Pickel, S. 2019; Pickel und Yendell 2018) verweisen auf den hohen Bedeutungsgehalt antimuslimischer Ressentiments und der Ablehnung von Migration nach Deutschland für die Wahl der rechtspopulistischen und rechtsradikalen AfD (Tab. 8). Unsere Befunde deuten auch für Berlin in diese Richtung. Erneut kommt den Bedrohungsängsten eine zentrale Bedeutung zu. Der Effekt der gefühlten Bedrohung vor Muslim*innen übertrifft alternative Erklärungsmodelle, wie z.B. fehlende soziale Anerkennung, relative Deprivation und wirtschaftliche Deprivationslagen (Manow 2018). AfD-Wähler*innen in Berlin wählen ihre Partei vor allem aufgrund einer autoritären Einstellung und einer politisch-ideologischen Selbsteinordnung als rechts. Diese Effekte werden gestützt durch Bedrohungsängste gegenüber Geflüchteten und Muslim*innen sowie dem Hang zu einer Verschwörungsmentalität. Nimmt man die Bedrohungsängste aus dem Modell, so gewinnen Vorurteile gegenüber den entsprechenden Gruppen an Relevanz, auch wenn die Gesamterklärungskraft des Modells leicht sinkt. Dies spricht für einen eigenständigen Effekt der Bedrohungsängste und einen gleichzeitigen Mediatoreffekt für bestehende Vorurteile durch die Äußerung von Bedrohungsängsten. Zudem passt dieses Bild einer Verselbständigung von Bedrohungsängsten, die man zur Erklärung der Abgrenzung zu einer sozialen Gruppe heranzieht perfekt in die Überlegungen der Integrated Threat Theory und der Social Identity Theory. Beide Theorien markieren dies als einen sich wechselseitig stärkenden und verstärkenden Prozess, der die Abgrenzung etabliert und bestärkt. Die Konstruktion der Bedrohung und ihre andauernde mediale Visibilisierung und Mobilisierung durch die AfD und ihre Politiker*innen tut ihr übriges für diesen Prozess. ${ }^{32}$

\section{Fazit: Religiöse Gemeinschaften als Bedrohungsszenario und Push- Faktor für die Wahl von Rechtspopulis*innen - auch in Berlin!}

Die Ergebnisse des Berlin-Monitors zeigen, selbst wenn die Werte in Berlin zumeist unter vergleichbaren Werten im Bundesgebiet liegen (Pickel et al. 2019), finden sich auch in Berlin Rassist*innen, Antisemit*innen und Muslimfeind*innen. Allerdings drückt sich der Status von Berlin als multikulturelle Stadt, mit einer Vielzahl von interethnischen und interreligiösen Kontaktmöglichkeiten in einer vergleichsweise moderaten Ausprägung von gruppenbezogenen Vorurteilen und Ressentiments aus. Diese Beschreibung trifft auch die Abwertung religiöser Gruppen. In ihrer multikulturellen Zusammensetzung nimmt Berlin eine gewisse Sonderstellung in Deutschland ein, ist aber auch in Teilen prototypisch für moderne Stadtgesellschaften in Demokratien. So wie es gruppenbezogene Vorurteile gegenüber vielen Gruppen in der Gesellschaft gibt, fallen diese - angesichts der Differenzen der verwendeten Items mit Vorsicht interpretiert - unterschiedlich stark aus. Zwar finden sich

32 Die Erklärungsmodelle sind immer zurückhaltend und eingedenk der speziellen Berliner Situation zu interpretieren. 
Personen, welche aufgrund ihrer gebündelten Ablehnung gleich mehrerer sozialer Gruppen als menschenfeindlich im Sinne der Gruppenbezogenen Menschenfeindlichkeit eingestuft werden können, allerdings existieren beachtliche Gruppengrößen in der Berliner Bevölkerung, die spezielle Vorurteile und Ressentiments aufweisen. So sind Vorurteile gegenüber Mitgliedern der islamischen Glaubensgemeinschaften nennenswert, wenn auch ein Minderheitenphänomen in Berlin.

$\mathrm{Zu}$ diesen Vorurteilen trägt unter vielen Berliner*innen neben rechter Ideologie, autoritären Einstellungen und übergreifendem Rassismus auch das Gefühl einer eigenen Bedrohung durch diese Gruppe bei. Folgt man weiteren Forschungsergebnissen, dann sehen einige Bürger*innen Muslim*innen aufgrund ihrer scheinbar ,aggressiven, gefährlichen und sich ausbreitenden“ Religion als eine Bedrohung der eigenen Ordnung und der eigenen Gesellschaft an (auch Pollack et al. 2014; Pickel und Yendell 2016). Stichworte wie „Überfremdung“ oder „Umvolkung“ machen hier die Runde. Dabei verbinden sich realistische mit symbolischen Bedrohungswahrnehmungen zu einem unguten Gefühl gegenüber Muslim*innen und „,dem Islam“. Auch gegenüber Jüd*innen existiert bei einigen - in Berlin wenigen - Bürger*innen das Gefühl einer Bedrohung. Diese Bedrohung scheint aber - so unsere Interpretation - eher Konsequenz tiefer liegender Verschwörungstheorien zu sein, als Folge eines aktuellen Bedrohungsgefühls. Alte Verschwörungstheorien des ,,jüdischen Strebens nach Weltherrschaft" befördern antisemitische Einstellungen und nicht zuletzt antisemitische Handlungen. Teilweise laufen die verschiedenen Vorurteile sogar zusammen, werden doch Jüd*innen als Drahtzieher hinter dem von Rechtspopulist*innen propagierten ,großen Austausch“, durch die forcierte Zuwanderung von Muslim*innen, gesehen. Autoritäre Einstellungen befördern eine solche Sicht genauso, wie eine formal geringe Bildung oder ein höheres Lebensalter. Da die vorherrschenden Bedrohungsgefühle oft nicht als Reaktion auf echte Erfahrungen oder Bedrohungslagen einzuschätzen sind, und zudem nur eine Erklärung neben grundsätzlicheren Erklärungsmustern darstellen, sind die Überlegungen der Integrated Threat Theory der eigenen Gruppenabschottung als Interpretation funktionstüchtig und keineswegs als Entschuldigung für Vorurteile einzustufen. So wie bei einem Teil derjenigen, welche Vorurteile gegenüber Muslim*innen äußern, tatsächlich Ängste für die Vorurteile bedeutsam sind, betonen andere die Bedrohungen aus einem tiefer liegendem Ressentiment, wenn nicht Rassismus heraus.

Und so ergeben sich - zumindest in Berlin - funktionale Unterschiede. Während antimuslimische Ressentiments weitgehend aus einer Kombinatorik von kulturellem Bedrohungsszenario, Überfremdungsangst und rechter Gesinnung getragen wird, besitzt der Antisemitismus auf allen Ebenen zwei unterschiedliche Einflussfaktoren, oder Gruppen. Zum einen sind antisemitische Einstellungen unter rechtsorientierten Verschwörungstheoretikern stark verbreitet. Sie glauben noch immer an eine jüdische Weltverschwörung und kombinieren dies mit Schuldabwehrmechanismen und Umwegkommunikation. Diese Haltung ist insbesondere im rechten Spektrum der politischen Landschaft stark verbreitet und stellt den wichtigsten Faktor für antisemitische Einstellungen dar. Daneben existiert aber auch eine andere Triebkraft für Antisemitismus: Dieser gründet in einer schwer zu entflechtenden Mischung aus Antijudaismus, israelbezogenen Antisemitismus und Herkunftserfahrungen von Muslim*innen. Speziell das häufigere Auftreten dogmatischer Vorstellungen unter 
den Berliner Muslim*innen erweist sich als begünstigend für die Ausprägung antisemitischer Einstellungen. Dies ist kein zwingender Zusammenhang und gläubige Muslim*innen sollten keineswegs unter den Generalverdacht eines muslimischen Antisemitismus fallen, gleichwohl scheint die Anfälligkeit in dieser sozialen Gruppe signifikant höher als in anderen Gruppen (höchstens noch vergleichbar mit der geringeren Zahl an christlichen Dogmatikern).

Die Existenz gruppenbezogener Vorurteile ist aus Sicht einer Perspektive einer demokratischen politischen Kultur alles andere als nebensächlich. Gruppenbezogenen Vorurteile, wenn sie vorhanden sind, erzeugen eine politische Wirkung. Ressentiments gegenüber Muslim*innen und Ängste ihnen gegenüber und Geflüchteten gegenüber sind ein zentrales Vehikel für Wahlerfolge rechtspopulistischer Parteien in Europa, wie es in Deutschland die $A f D$ ist. Unter AfD-Anhänger*innen sind fast alle gruppenbezogenen Vorurteile und Ressentiments signifikant stärker als in anderen Teilen der Berliner Bevölkerung. Dies gilt speziell für die auf Muslim*innen und Jüd*innen bezogenen Ressentiments wie für die gefühlte Bedrohung seitens dieser Religionsgemeinschaften. In multivariaten Analysemodellen dominiert dann auch die Angst vor Muslim*innen und Geflüchteten und überdeckt die meisten anderen, intersektional vorhandenen, Vorurteile. Konkret gesagt: Bedrohungsgefühle und Ressentiments spielen eine beachtliche Rolle für die Wahl der AfD - in Berlin. Damit kann man nicht sagen, dass antipluralistische bis demokratiefeindliche Haltungen reines Merkmal der AfD-Spitze sind, während die Wählerschaft weitgehend aus protestorientierten Bürger*innen besteht. Letztere mögen durchaus Wähler*innen der AfD sein, sie teilen sich ihr Wahlverhalten aber mit überdurchschnittlich muslimfeindlichen Wähler*innen.

Die Angst vor religiösen Gruppen scheint eine zentrale Kraft unter den gruppenbezogenen Vorurteilen und für deren Auswirkungen auf die Haltung zur Demokratie und das Wahlverhalten zu sein. Und dies geht über die damit verbundene Verletzung des Grundrechts auf Religionsfreiheit hinaus. Die Zugehörigkeit zu religiöse Gruppen ist gut zu identifizieren und zu klassifizieren, was ein Bestandteil der Vorurteilsbildung ist. Sie sind vom Hauch der auch durch Umfeldbedingungen nicht veränderbaren Kulturalität umweht und eignen sich gut für ein Feindbild mit Bedrohungspotential. Dies unterscheidet Vorurteile und Ressentiments gegenüber Muslim*innen und Jüd*innen von anderen Vorurteilen: Sie sie nicht unbedingt weiter verbreitet, besitzen aber eine stärkere Wirkung auf antidemokratische Einstellungen und rechtspopulistische Mobilisierungserfolge. Zudem sind sie gut mit Verschwörungstheorien zu verknüpfen. Man kann sagen: Religion matters, aber etwas anders als man sich dies vielleicht wünschen würde.

Funding Open Access funding provided by Projekt DEAL.

Open Access Dieser Artikel wird unter der Creative Commons Namensnennung 4.0 International Lizenz veröffentlicht, welche die Nutzung, Vervielfältigung, Bearbeitung, Verbreitung und Wiedergabe in jeglichem Medium und Format erlaubt, sofern Sie den/die ursprünglichen Autor(en) und die Quelle ordnungsgemäß nennen, einen Link zur Creative Commons Lizenz beifügen und angeben, ob Änderungen vorgenommen wurden.

Die in diesem Artikel enthaltenen Bilder und sonstiges Drittmaterial unterliegen ebenfalls der genannten Creative Commons Lizenz, sofern sich aus der Abbildungslegende nichts anderes ergibt. Sofern das betref- 
fende Material nicht unter der genannten Creative Commons Lizenz steht und die betreffende Handlung nicht nach gesetzlichen Vorschriften erlaubt ist, ist für die oben aufgeführten Weiterverwendungen des Materials die Einwilligung des jeweiligen Rechteinhabers einzuholen.

Weitere Details zur Lizenz entnehmen Sie bitte der Lizenzinformation auf http://creativecommons.org/ licenses/by/4.0/deed.de.

\section{Literatur}

Adida, Claire, David D. Laitin, und Marie-Anne Valfort. 2016. Why muslim integration fails in Christianheritage societies. Cambridge: Harvard University Press.

Adorno, Theodor W. 1973. Studien zum autoritären Charakter, 1. Aufl., Frankfurt/Main: Suhrkamp. Erstveröffentlichkeit: 1950.

Allport, Gordon W. 1979. The nature of prejudice, 3. Aufl., New York: Basic Books. Erstveröffentlichung 1954.

Allport, Gordon W., und Michael J. Ross. 1967. Personal religious orientation and prejudice. Journal of Personality and Social Psychology 5(4):432-435.

Almond, Gabriel, und Sidney Verba. 1963. The civic culture. Political attitudes and democracy in five nations. Princeton: Princeton University Press.

Bas, Yasin. 2019. Muslime in den Medien. Presseschau 2018. Köln.

Beller, Steven. 2015. Antisemitism. A Very Short Introduction. Oxford: Oxford University Press.

Beyer, Heiko, und Ivar Krumpal. 2010. „Aber es gibt keine Antisemiten mehr“: Eine experimentelle Studie zur Kommunikationslatenz antisemitischer Einstellungen. Kölner Zeitschrift für Soziologe und Sozialpsychologie 39:215-232.

Blalock, Hubert M., Jr.. 1967. Toward a theory of minority group relations. New York: John Wiley.

Bleich, Erik. 2011. What is Islamophobia. And how much is there? Theorizing and measuring an emerging comparative concept. American Behavioral Scientist 55:1581-1600.

Blume, Michael. 2019. Warum der Antisemitismus uns alle bedroht. Wie neue Medien alte Verschwörungsmythen befeuern. Ostfildern: Patmos.

Blumer, Herbert G. 1958. Race prejudice as a sense of group position. Pacific Sociological Review 1(1):3-7.

Brown, Rupert, und Dominik Abrams. 1986. The effects of ingroup similarity and goal interdependence attitudes and task performance. Journal of Experimental Social Psychology 22(1):78-94.

Cakir, Naime. 2014. Islamfeindlichkeit. Anatomie eines Feindbildes in Deutschland. Bielefeld: transkript.

Carol, Sarah, Marc Helbling, und Ines Michalowski. 2015. A struggle over religious rights? How Muslim minorities and natives view the accommodation of religion in six European countries. Social Forces 94(2):647-671.

Castanho Silva, Bruno, Sebastian Jungkunz, und Marc Helbling. 2019. A empirical comparison of seven populist attitude scales. Political Research Quarterly 72:1-16. https://doi.org/10.1177/ 1065912919833176.

Decker, Oliver, und Elmar Brähler. 2018. Flucht ins Autoritäre: Rechtsextreme Dynamiken in der Mitte der Gesellschaft. Gießen: Psychosozial.

Decker, Oliver, und Christoph Türcke (Hrsg.). 2019. Autoritarismus. Kritische Theorie und Psychoanalytische Praxis. Gießen: Psychosozial.

Decker, Oliver, Johannes Kiess, und Elmar Brähler. 2016. Die enthemmte Mitte. Autoritäre und rechtsextreme Einstellungen in Deutschland. Gießen: Psychosozial.

Decker, Oliver, Johannes Kiess, und Elmar Brähler. 2018. Antisemitische Ressentiments in Deutschland Verbreitung und Ursachen. In Flucht ins Autoritäre - autoritäre und rechtsextreme Dynamiken in der Mitte der Gesellschaft, Hrsg. Oliver Decker, Elmar Brähler, 141-178. Gießen: Psychosozial.

Diangelo, Robin. 2018. White Fragility. Why it's so hard for White People to Talk About Racism. Boston: Beacon Press.

Easton, David. 1975. A re-assessment of the concept of political support. British Journal of Political Science 5:435-457.

Fiske, Susan, Amy Cuddy, Peter Glick, und Jun Xu. 2002. A model of (often mixed) stereotype content: competence and warmth respectively follow from perceived status and competition. Journal of Personality and Social Psychology 82:878-902.

Forst, Rainer. 2003. Toleranz im Konflikt. Geschichte, Gehalt und Gegenwart eines umstrittenen Begriffs. Frankfurt/Main: Suhrkamp. 
Frinte, Wolfgang und Dieter Wammelberger. 2008. Antisemitismus, Israelkritik, Nationalismus - Empirische Befunde. Berliner Debatte Initial 19:29-42.

Gonzalez, Karina Velasco, Maykel Verkuyten, Jeroen Weesie, und Edwin Poppe. 2008. Prejudice towards muslims in the Netherlands: testing integrated threat theory. British Journal of Social Psychology 47:667-685.

Hafez, Kai, und Sabrina Schmidt. 2015. Die Wahrnehmung des Islam in Deutschland. Gütersloh: Bertelsmann.

Halliday, Fred. 1999. Islamophobia reconsidered. Ethnic and Racial Studies 22:892-902.

Hambauer, Verena, und Anja Mays. 2018. Wer wählt die AfD? - Ein Vergleich zwischen Sozialstruktur, politischen Einstellungen und Einstellungen zu Flüchtlingen zwischen AfD-WählerInnen und WählerInnen anderer Parteien. Zeitschrift für Vergleichende Politikwissenschaft (ZfVP) 12(1):133-154. Special Issue: Migration und Integration als politische Herausforderung - Vergleichende Analysen zu politisch-kulturellen Voraussetzungen der Migrationspolitik und Reaktionen.

Hasters, Alice. 2019. Was weiße Menschen nicht über Rassismus hören wollen. München: hanserblau.

Heitmeyer, Wilhelm. 2002. Gruppenbezogene Menschenfeindlichkeit. Die theoretische Konzeption und erste empirische Ergebnisse. In Deutsche Zustände. Folge I, Hrsg. Wilhelm Heitmeyer, 15-34. Frankfurt/M.: Suhrkamp.

Heitmeyer, Wilhelm. 2010. Deutsche Zustände. Folge 10. Frankfurt a.M.: Suhrkamp.

Heitmeyer, Wilhelm. 2018. Autoritäre Versuchungen. Frankfurt/Main: Suhrkamp.

Helbling, Marc (Hrsg.). 2012. Islamophobia in the west: measuring and explaining individual attitudes. London: Routledge.

Hewstone, Miles, und Hermann Swart. 2011. Fifty-odd years of inter-group contact: From hypothesis to integrated theory. British Journal of Social Psychology 50:374-386.

Hirschmann, Albert O. 1970. Exit, voice and loyality. Responses to decline in firms, organizations and states. Cambridge: University Press.

Horton, Donald, und Richard R. Wohl. 1956. Mass communication and para-social interaction: observations on intimacy at a distance. Psychiatry 19:215-229.

Huntington, Samuel P. 1996. Kampf der Kulturen. Die Neugestaltung der Weltpolitik im 21. Jahrhundert. Wien: Europa Verlag.

Jonas, Eva, und Immo Fritsche. 2013. Destined to die but not to wage war. How existential threat can contribute to escalation or de-escalation of violent intergroup conflict. Amercian Psychologist 68(7):543-558.

Jonas, Klaus, Wolfgang Stroebe, und Miles Hewstone. 2014. Sozialpsychologie, 6. Aufl., Heidelberg: Springer.

Klein, Anna. 2014. Toleranz und Vorurteil. Zum Verhältnis von Toleranz und Wertschätzung zu Vorurteilen und Diskriminierung. Opladen: Barbara Budrich.

Koopmans, Ruud. 2015. Religious fundamentalism and hostility against out-groups: a comparison of muslims and christians in western europe. Journal of Ethnic and Migration Studies 41(1):33-57.

Koopmans, Ruud. 2017. Assimlation oder Multikulturalismus? Bedingungen gelungener Integration. Berlin: Lit Verlag.

Koopmans, Ruud. 2020. Das verfallene Haus des Islam. Die religiösen Ursachen von Unfreiheit, Stagnation und Gewalt. München: C.H. Beck.

Leibold, Jürgen, und Steffen Kühnel. 2003. Islamophobie. Sensible Aufmerksamkeit für spannungsreiche Anzeichen. In Deutsche Zustände 2, Hrsg. Wilhelm Heitmeyer, 100-119. Frankfurt/Main: Suhrkamp.

Liedhegener, Antonius. 2016. Religiöse Identitäten als Problem wechselseitiger Identifizierungen und Kategorisierungen. Aktuelle theoretische Konzepte und Fragen ihrer Operationalisierung in der empirischen Religionsforschung. In Religiöse Identitäten in politischen Konflikten, Hrsg. Ines-Jacqueline Werkner, Oliver Hidalgo, 65-82. Wiesbaden: Springer VS.

Lipset, Seymour M. 1981. Political man: the social bases of politics. Baltimore: Johns Hopkins.

Lipstadt, Deborah. 2019. Der neue Antisemitismus. Berlin: Berlin Verlag.

Manow, Philip. 2018. Die politische Ökonomie des Populismus. Frankfurt/Main: Suhrkamp.

Memmi, Albert. 1987. Rassismus. Königstein: Athenäum.

Mudde, Cas, und Christobal Rovira Kaltwasser. 2017. Populism. A very short introduction. Oxford: University Press.

Muno, Wolfgang. 2009. Fallstudien und die Vergleichende Methode. In In Methoden der vergleichenden Politik- und Sozialwissenschaft. Neue Entwicklungen und Anwendungen, Hrsg. Susanne Pickel, Gert Pickel, Hans-Joachim Lauth, und Detlef Jahn, 113-132. Wiesbaden: VS.

Parsons, Talcott. 1951. The social system. New York: Free Press.

Pelinka, Anton, Karin Bischof, und Karin Stöger. 2009. Handbook of prejudice. New York: Cambria Press. 
Pettigrew, Thomas F. 1958. Personality and sociocultural factors in intergroup attitudes: A cross-national comparison. Journal of Conflict Resolution 2:29-42.

Pettigrew, Thomas F. 1998. Intergroup contact theory. Annual Review of Psychology 49:65-85.

Pettigrew, Thomas F., und Linda Tropp. 2006. A meta-analytic test of intergroup contact theory. Journal of Personality and Social Psychology 90:751-783.

Pickel, Gert. 2018. Religion als Ressource für Rechtspopulismus? Zwischen Wahlverwandtschaften und Fremdzuschreibungen. Zeitschrift für Religion, Gesellschaft und Politik 2(2):277-312. https://doi.org/ 10.1007/s41682-018-0032-z.

Pickel, Susanne. 2019. Die Wahl der AfD. Frustration, Deprivation, Angst oder Wertekonflikt? In Die Bundestagswahlen 2017, Hrsg. Karl-Rudolf Korte. Wiesbaden: Springer VS.

Pickel, Gert, und Cemal Öztürk. 2018. Islamophobia without muslims? The "contact hypothesis" as an explanation for anti-muslim attitudes - eastern European societies in a comparative perspective. Journal of Nationalism, Memory \& Language Politics 12(2):162-191.

Pickel, Susanne, und Gert Pickel. 2006. Politische Kultur- und Demokratieforschung. Grundbegriffe, Theorien, Methoden. Eine Einführung. Wiesbaden: VS.

Pickel, Gert, und Susanne Pickel. 2018. Migration als Gefahr für die politische Kultur? Kollektive Identitäten und Religionszugehörigkeit als Herausforderung demokratischer Gemeinschaften. Zeitschrift für Vergleichende Politikwissenschaft (ZfVP) 12(1):297-320. Special Issue: Migration und Integration als politische Herausforderung - Vergleichende Analysen zu politisch-kulturellen Voraussetzungen der Migrationspolitik und Reaktionen.

Pickel, Gert, und Susanne Pickel. 2019. Der Flüchtling als Muslim - und unerwünschter Mitbürger? In Flucht und Migration in Europa. Neue Herausforderungen für Parteien, Kirchen und Religionsgemeinschaften, Hrsg. Oliver Hidalgo, Gert Pickel, 279-324. Wiesbaden: Springer VS.

Pickel, Susanne, und Gert Pickel. 2020a. Politische Kulturforschung. Eine Einführung. Wiesbaden: Springer VS.

Pickel, Gert und Susanne Pickel. 2020b. Gesellschaftlicher Zusammenhalt (in Sachsen) als Konsequenz von Sozialkapital, Responsivität und positiver Transformationsverarbeitung. In: Sachsen zwischen Integration und Desintegration. Politisch-kulturelle Heimaten, Hrsg. Steffen Kailitz, Frank Asbrock, Heike Greschke, Gert Pickel, Sheryn Rindermann, Antje Röder und Julia Schulze-Wessel. Wiesbaden: Springer VS. (i.E.).

Pickel, Gert, und Alexander Yendell. 2016. Islam als Bedrohung? Beschreibung und Erklärung von Einstellungen zum Islam im Ländervergleich. Zeitschrift für Vergleichende Politikwissenschaft 10(3-4):273-309.

Pickel, Gert, und Alexander Yendell. 2018. Religion als konfliktärer Faktor in Zusammenhang mit Rechtsextremismus, Muslimfeindschaft und AfD-Wahl. In Flucht ins Autoritäre. Rechtsextreme Dynamiken in der Mitte der Gesellschaft, Hrsg. Oliver Decker, Elmar Brähler, 217-243. Gießen: Psychosozial.

Pickel, Gert, Alexander Yendell, und Yvonne Jaeckel. 2016. Religiöse Pluralität als Bedrohung oder kulturelle Bereicherung? Die Wahrnehmung von Bedrohung durch Religion im Ländervergleich. In Religiöse Identitäten in politischen Konflikten, Hrsg. Ines-Jacqueline Werkner, Oliver Hidalgo, 83-123. Wiesbaden: Springer VS.

Pickel, Gert, Katrin Reimer-Gordinskaya, und Oliver Decker (Hrsg.). 2019. Berlin-Monitor 2019. Vernetzte Solidarität - Fragmentierte Demokratie. Springe: zu Klampen.

Pickel, Gert, Antonius Liedhegener, Anastas Odermatt, Yvonne Jaeckel, und Alexander Yendell. 2020. Religiöse Identitäten und Vorurteil in Deutschland und der Schweiz - Konzeptionelle Überlegungen und empirische Befunde. Zeitschrift für Religion, Gesellschaft und Politik. https://doi.org/10.1007/ s41682-020-00055-9.

Pollack, Detlef, Olaf Müller, Gergely Rosta, Nils Friedrichs, und Alexander Yendell. 2014. Grenzen der Toleranz: Wahrnehmung und Akzeptanz religiöser Vielfalt in Europa. Wiesbaden: Springer VS.

Putnam, Robert. 2000. Bowling Alone. The Collapse and Revival of American Community. New York: Simon and Schuster.

Quendt, Matthias. 2019. Deutschland rechts außen. Wie die Rechten nah der Macht greifen und wie wir sie stoppen können. München: Piper.

Quillian, Lincoln. 1995. Prejudice as a response to perceived group threat. Population composition and anti-immigrant and racial prejudice in Europe. American Sociological Review 60:586-611.

Ranc, Johannes. 2016. Eventuell nicht gewollter Antisemitismus. Zur Kommunikation antijüdischer Ressentiments unter Deutschen Durchschnittsbürge. Münster: Westfälisches Dampfboot.

Rees, Jonas, und Pia Lamberty. 2019. Mitreißende Wahrheiten: Verschwörungsmythen als Gefahr für den gesellschaftlichen Zusammenhalt. In Verlorene Mitte - Feindselige Zustände. Rechtsextreme Einstel- 
lungen in Deutschland 2018/19, Hrsg. Andreas Zick, Beate Küpper, und Wilhelm Berghan, 203-222. Bonn: Dietz.

RIAS (Recherche und Informationsstelle Antisemitismus, Berlin). 2019. Antisemitische Vorfälle 2018. Bericht der Recherche- und Informationsstelle Antisemitismus. Berlin: Verein für Demokratische Kultur in Berlin.

Rippl, Susanne, und Christian Seipel. 2018. Modernisierungsverlierer, Cultural Backlash, Postdemokratie. Was erklärt rechtspopulistische Orientierungen? Kölner Zeitschrift für Soziologie und Sozialpsychologie https://doi.org/10.1007/s11577-018-0522-1.

Salzborn, Samuel. 2014. Antisemitismus. Geschichte, Theorie, Empirie. Baden-Baden: Nomos.

Schlueter, Elmar, und Peer Scheepers. 2010. The relationship between outgroup size and anti-outgroup attitudes: A theoretical synthesis and empirical test of group threat- and intergroup contact theory. Social Science Research 39(2):285-295.

Schönbach, Peter. 1961. Reaktionen auf die antisemitische Welle im Winter 1959/1960. Frankfurt/Main: Europäische Verlagsanstalt.

Sherif, Muzafer, Otis J. Harvey, Jack White, William Hood, und Carolyn Sherif. 1961. The robbers cave experiment. Intergroup conflict and cooperation. Norman: University of Oklahoma Books.

Shooman, Yasemin. 2014. weil ihre Kultur so ist 《〈. Narrative des antimuslimischen Rassismus. Bielefeld: transkript.

Stephan, Walter G., und Cookie W. Stephan. 1996. Predicting prejustice. International Journal of Intercultural Relations 20:409-426.

Stephan, Walter G., und Lausanne C. Renfro. 2002. The role of threat in intergroup relations. In From prejudice to intergroup emotions: differentiated reactions to social groups, Hrsg. Diane M. Mackie, Elliot R. Smith, 191-207. New York: Psychology Press.

Stephan, Walter G., Rolando Diaz-Loving, und Anne Duran. 2000. Integrated threat theory and intercultural attitudes-Mexico and the United States. Journal of Cross-Cultural Psychology 31(2):240-249.

Strabac, Zan, und Ola Listhaug. 2007. Anti-Muslim prejudice in Europe: a multilevel analysis of survey data from 30 countries. Social Science Research 37:268-286.

Tajfel, Henri. 1982. Social identity and intergroup relations. Cambridge: University Press.

Tajfel, Henri, und John C. Turner. 1986. The social identity theory of intergroup behavior. In The social psychology of intergroup relations, Hrsg. Stephan Worchel, William Austin, 7-24. Chicago: NelsonHall.

Tönnies, Ferdinand. 2010. Gemeinschaft und Gesellschaft. Grundbegriffe der reinen Soziologie. Darmstadt: Wissenschaftliche Buchgesellschaft. Erstausgabe: 1887.

Uenal, Fatith. 2016. Disentangling Islamophobia: the differential effects of symbolic, realistic, and terroristic threat perceptions as mediators between social dominance orientation and Islamophobia. Journal of Social and Political Psychology 4(1):66-90.

Yendell, Alexander. 2013. Muslime unerwünscht? Zur Akzeptanz des Islam und dessen Angehörigen. Ein Vergleich zwischen west- und Ostdeutschland. In Religion und Politik im vereinigten Deutschland. Was bleibt von der Rückkehr des Religiösen?, Hrsg. Gert Pickel, Oliver Hidalgo, 221-248. Wiesbaden: Springer VS.

Zick, Andreas, und Anna Klein. 2014. Fragile Mitte - Feindselige Zustände. Rechtsextreme Einstellungen in Deutschland 2014. Bonn: Dietz.

Zick, Andreas, und Beate Küpper. 2009. Attitudes towards the Islam and Muslims in Europe. Bielefeld: Short Report.

Zick, Andreas, Beate Küpper, und Andreas Hövermann. 2011. Die Abwertung des Anderen. Eine europäische Zustandsbeschreibung zu Intoleranz, Vorurteilen und Diskriminierung. Berlin: Friedrich-Ebert Stiftung.

Zick, Andreas, Beate Küpper, und Daniela Krause. 2016. Gespaltene Mitte-Feindselige Zustände. Rechtsextreme Einstellungen in Deutschland 2016. Bonn: Dietz.

Zick, Andreas, Beate Küpper, und Wilhelm Berghan. 2019. Verlorene Mitte - Feindselige Zustände. Rechtsextreme Einstellungen in Deutschland 2018/19. Bonn: Dietz. 